\title{
MONTHLY
}

JAMES E. CASKEY, JR., Editor

\section{ON THE THEORY OF DISTURBANCES IN A CONDITIONALLY UNSTABLE ATMOSPHERE}

\author{
DOUGLAS K. LILLY \\ General Circulation Research Section, U.S. Weather Bureau, Washington, D.C. \\ [Manuscript received November 2, 1959; revised January 22, 1960]
}

\begin{abstract}
A perturbation model is developed which is applicable to small-amplitude moist convective disturbances of scales ranging from those of squall lines to tropical cyclones. In an extension of the works of Syōno and Haque, disturbance development is studied for an atmosphere in which static stability may vary with height and with the sense of the vertical motion, being in general negative for upward motion in some substantial layer. Closed solutions for simplified cases are exhibited as well as results of numerical integration of a more realistic case. The dynamic stability criterion depends on the static stabilities in the ascending and descending currents and their dimensions and geometric relationships. Cloud-scale and meso-scale disturbances are more unstable than those of cyclone-scale, and presence of the former may tend to destroy conditions favoring development of the latter. Evaluation is made of the linearized effects of parallel convective bands, an "eye", the tropopause, nonhydrostatic motions, surface friction, and various boundary conditions. Comparison of solutions with observational features shows fair agreement in some respects, improving considerably when nonlinear effects are qualitatively considered. The disturbances are effective in transporting kinetic and potential energy outward from the actively unstable updraft. Nonlinear interactions tend to transport heat upward to modify the initial static stability distribution. A second-order dynamic stability criterion, obtained by consideration of nonlinear effects, tends to favor development of an existing finite-amplitude disturbance of tropical cyclone scale.
\end{abstract}

\section{INTRODUCTION}

One of the most successful mathematical tools for the study and elucidation of meteorological phenomena has been the linearized perturbation model. Our present understanding of synoptic-scale motions in the troposphere is, in fact, strongly dependent upon studies of linearized barotropic and baroclinic motions and these also have been necessary prerequisites to construction of useful nonlinear forecasting models. In light of this backgroind it would seem that the study of linearized moist convection might provide a useful background to attempts at dynamical forecasts for regions and scales of motion for which such phenomena are important.
"Dry" convective motions, i.e., those involving no changes of phase, have been studied theoretically and observationally for many years. Results of linear theory-the stability criterion including the effects of scale, symmetry, and the fluid parameters-have been fairly well verified in the special cases where the assumptions of the theory hold. The importance and complexity of the phenomenon are such that it still provides a fertile field for investigation, particularly in regard to nonlinear effects. It seems likely, however, that many important forms of atmospheric convection cannot be considered as special cases of Bénard cellular convection. In particular, the largest important form of direct convective circulation, the tropical cyclone, develops in considerable contradic- 
tion to the scale selectivity results of Bénard cell theory. While this contradiction has never been adequately resolved, it is perhaps generally agreed that consideration of condensation effects is indispensable to its satisfactory explanation.

In recent years two authors have considered linear solutions of a complete nonviscous set of hydrodynamic equations with inclusion of condensation effects; in both cases the results were applied principally to tropical cyclogenesis. Syōno [21] considered a frictionless quasistatic system with static stability negative for all vertical motions, and allowing a certain very restrictive form of basic flow obtained solutions similar to those of type I of this investigation. Haque [8] considered motions on a basic rest state, but allowed the static stability to change sign with the sense of the vertical motion, to approximate the effects of moist updraft and dry downdraft. He also used the nonhydrostatic vertical equation of motion and considered the effects of an "eye" and (somewhat erroneously) the tropopause. In both these studies there were certain deficiencies in the physical interpretation of the results. In particular, no evident attempt was made to reconcile the stability criterion, which indicated complete dominance of cloud-scale systems, with the actual existence and growth of disturbances of much greater time and space scales. There was no consideration given to the possibility of line-symmetric disturbances, or of circular convective bands about a center. Finally the energy transports and transformations were not discussed and no mention was made of nonlinear effects, even as to orders of magnitude.

This study may be considered to be, in large part, an extension of the results of Syōno and Haque in an attempt to rectify the deficiencies mentioned above, and to include as well cursory investigations of the effects of surface friction, some variation of boundary conditions, and realistic distribution of static stability for both moist and dry processes. It appears that there is also some applicability of linear methods to meso-scale convection; e.g., that of cloud bands, as distinguished from individual cloud cells. It was therefore considered desirable to develop some cases pertaining to meso-scale motions and their interrelation with those of larger scale.

A further purpose of this study should be mentioned, one which has particular significance to the field of numerical weather forecasting. Recent results of Phillips [19] and Smagorinsky [20] lead to the conclusion that a forecast scheme using the primitive equations of motion is practical and in several respects more satisfactory for prediction of baroclinic motions than schemes using derived equations. If such a scheme is used in a model in which moisture and its thermodynamic effects are included explicitly and static stability is self-determined, then one may anticipate the occasional development of conditionally unstable inertio-gravitational motions, which may grow to large amplitudes unless suppressed by some more-or-less arbitrary method. The conclusions of this study indicate that meso-scale and cloud-scale convecti are generally sufficient physical mechanisms for release this instability. Under certain special initial conditic however, larger scale motions may develop, whose dim sions are determined by both the linear and nonlin terms of the dynamic equations.

\section{PLAN OF INVESTIGATION}

As stated in the introduction, the principal results this study will be obtained by use of the linearized atr pheric equations. The effects of nonlinear terms $v$ however, be considered in assessing the results and valic of the linear theory and for obtaining second-order , rections to these results. The variation of Cori parameter with latitude and the possible existence $c$ nonuniform basic flow will be ignored, as well as the effi of nonuniform orography. It is found possible, howe to investigate some of the effects of surface friction, tropopause, and acceleration terms in the vertical eq tion of motion. Some of the most significant results this investigation are found upon consideration of $m$ convective elements surrounded by or scattered throug nonsaturated air mass.

The investigation proceeds as follows: The basic lir nonviscous differential equations in the $p$-coordinate : tem are reduced to a single fourth-order partial diffes tial equation with $\omega$ as the dependent variable. Bound and initial conditions are constructed from appropr physical considerations. Eigenfunction solutions obtained in time, space, and pressure for varying sp fications of hydrostatic stability and boundary conditi Eigenvalue considerations lead to a wave equation re ing the horizontal and vertical disturbance scale par: eters, hydrostatic stability, and rate of disturbance grow The wave equation is investigated to clarify the relat: ship of the factors determining growth rate. Correctj to the wave equation for nonhydrostatic terms in vertical equation of motion are discussed and the $c$ relationship of the solutions to internal gravitatic waves is pointed out.

The energy transformation and transport terms next considered, and special attention is paid to the trs fer of energy from active (moist) regions to adjoir passive (dry) regions.

Finally we investigate the nonlinear terms aris when the linear solutions are substituted into the $\mathrm{c}$ plete meteorological equations, in order to determine validity of the perturbation approach, to show the char in the form of the disturbance and in the stability crites produced by nonlinear terms, and to indicate effect: the disturbances upon their environment.

\section{DIFFERENTIAL EQUATIONS AND BOUNDARY CONDITIONS}

The linearized nonviscous quasi-static equations atmospheric motion, continuity, and heat may be writ 
with pressure as the vertical coordinate (see Eliassen [4]) as follows, where perturbations were assumed on a basic barotropic rest state:

$$
\begin{gathered}
\frac{\partial \mathrm{V}}{\partial t}+f \mathbf{k} \times \mathbf{V}+\nabla \Phi=0 \\
\frac{\partial \Phi}{\partial p}+\alpha=0 \\
\nabla \cdot \mathrm{V}+\frac{\partial \omega}{\partial p}=0 \\
\frac{\partial \alpha}{\partial t}-\sigma \omega=0
\end{gathered}
$$

where $V$ is the horizontal velocity, $\Phi$ the geopotential, $\alpha$ the specific volume, and $\omega=d p / d t$, the quantity analogous to vertical motion. The Coriolis parameter $f$ is assumed constant and the static stability analog $\sigma=-(\partial \bar{\alpha} / \partial p+\bar{\alpha} /$ $\kappa p$ ) is a function of the space variables only, where $\kappa$ is a polytropic coefficient, equal to $c_{p} / c_{v}$ in the case of dry motions. This set is reduced to a single equation in $\omega$ by the elimination of variables as follows: Equation (1) is operated upon successively by the horizontal divergence and vertical curl' component operators and the vorticity eliminated between the two resulting equations to yield an equation in divergence and $\Phi$. Divergence is eliminated in favor of vertical velocity by (3), and the result combined with (2) and (4) to eliminate $\alpha$ and $\Phi$ and obtain the desired equation in $\omega$.

$$
\left(\frac{\partial^{2}}{\partial t^{2}}+f^{2}\right) \frac{\partial^{2} \omega}{\partial p^{2}}+\nabla^{2}(\sigma \omega)=0 .
$$

The stability factor $\sigma$ is the most important determinant of both the physical and mathematical features of equation (5). By use of the hydrostatic equation and the equation of state we can represent this parameter as follows:

$$
\sigma=-\frac{\bar{\alpha}}{\bar{T}}\left(\frac{\partial \bar{T}}{\partial p}-\frac{\kappa-1}{\kappa} \frac{\bar{T}}{p}\right)=\frac{\bar{\alpha}}{p} \frac{\gamma_{a d}-\gamma}{\gamma_{a c}}
$$

where $\gamma_{u d}=\frac{g}{R} \frac{\kappa-1}{\kappa}$, the dry or moist adiabatic lapse rate, $\gamma_{a c}=g / R$, the autoconvective lapse rate, and $\gamma=-\partial \bar{T} / \partial z$, the actual atmospheric lapse rate. Values of $\sigma$ for both dry and moist motions have been calculated from virtual temperatures for the mean hurricane season tropical atmosphere recently published by Jordan [12], and are given in table 1 , where $\sigma_{d}$ is the dry and $\sigma_{w}$ the moist stability.

At levels above the tropopause, where $\bar{T} \approx$ constant, equation (6) shows that:

$$
\sigma \approx \frac{R \bar{T}}{p^{2}} \frac{\gamma_{a a}}{\gamma_{a s}} \approx \frac{R^{2} \bar{T}}{c_{p} p^{2}}
$$

and therefore $1 / \sigma \rightarrow 0$ as $p^{2}$.
The top and bottom boundary conditions for solutions of (5) required by physical considerations are, respectively

$$
\begin{aligned}
& \omega=0 \text { at } p=0 \\
& w=0 \text { at } z=0
\end{aligned}
$$

where $w$ is the vertical velocity.

Expansion and linearization of the second of these give the form

$$
\frac{\partial \Phi}{\partial t}+\bar{\alpha} \omega=0 \text { at } z=0
$$

The surface $z=0$ corresponds approximately to the pressure surface $p=p_{0}+\Phi\left(p_{0}\right) / \bar{\alpha}\left(p_{0}\right)$. By analogy with the simple treatment of waves on a fluid surface, however, we assume (10) to apply to a pressure surface, so that

$$
\frac{\partial \Phi}{\partial t}+\bar{\alpha} \omega=0 \text { at } p=p_{0} .
$$

A further simplification, which will be shown to introduce little error in solutions of the types considered in this study, consists of neglect of the first term of (11), reducing it to the form:

$$
\omega=0 \text { at } p=p_{0} .
$$

The latter is known in numerical forecasting to be a filtering condition for elimination of external gravitational waves (Hollman [10]).

For consideration of the effects of a tropopause it will be shown that (8) may be effectively applied at a pressure level $p_{\mathbf{H}}$, where $p_{\mathbf{H}}$ is slightly less than $p_{\mathrm{T}}$, the tropopause pressure.

\begin{tabular}{|c|c|c|}
\hline Pressure (mb.) & $\sigma_{d}\left(10^{-3} \mathrm{~m} \cdot 2 \mathrm{mb} \cdot-2 \mathrm{sec} \cdot-2\right)$ & $\sigma w\left(10^{-3} \mathrm{~m} .^{2} \mathrm{mb} .^{-2} \mathrm{sec}^{-2}\right)$ \\
\hline $\begin{array}{l}950 \\
80000 \\
850 \\
700 \\
650 \\
6000 \\
500 \\
450 \\
400 \\
350 \\
300\end{array}$ & $\begin{array}{r}6.04 \\
9.24 \\
12.95 \\
14.85 \\
16.87 \\
18.26 \\
20.7 \\
24.6 \\
28.2 \\
32.0 \\
36.7 \\
39.0 \\
39.3 \\
42.1 \\
43.3 \\
104.2 \\
164 . \\
344 . \\
788 . \\
1837 .\end{array}$ & $\begin{array}{r}-9.96 \\
-8.00 \\
-5.81 \\
-5.54 \\
-5.40 \\
-6.04 \\
-6.58 \\
-5.31 \\
-4.06 \\
-2.32 \\
-1.30 \\
4.02 \\
8.17 \\
17.92 \\
30.3 \\
96.9 \\
159 . \\
341 . \\
784 . \\
1837 .\end{array}$ \\
\hline
\end{tabular}

The effects of surface friction are accounted for, rather crudely, by assuming a forced vertical motion at the top of the friction layer, proportional to the geostrophic vorticity, that is

$$
\omega=B\left(\frac{p_{\mathrm{F}}-p_{0}}{f}\right) \nabla^{2} \Phi \text { at } p=p_{\mathrm{F}} \approx 900 \mathrm{mb}
$$

where $B$ is a nondimensional coefficient, perhaps a function of scale. Assuming an Ekman spiral velocity profile in the friction layer, Charney and Eliassen [3] have

TABLE 1.-Values of dry and moist static stability for the mean hurricane season tropical atmosphere 
shown that $B=(\sin 2 \psi) / 2$, where $\psi$ is the indraft angle at the surface. The Ekman spiral, however, is not commonly observed in the subcloud layer over the tropical oceans. It turns out that we do not need to know $B$ exactly, but only its sign and general order of magnitude. We assume, therefore that $|B|<1$ and that $B$ is positive for large scales of motion and negative for small scales (where friction opposes the normal indraft).

As to external lateral boundary conditions, in most cases we consider the atmosphere to be horizontally infinite, so that the only requirement is that the dependent variables remain finite over all space. In the one case in which lateral limitations are used, it is assumed that the velocity normal to the boundary vanishes.

It will later be shown that meaningful solutions of (5) exist for which the static stability parameter, $\sigma$, may be discontinuous in the horizontal and/or vertical. At the discontinuities the differential equations involving $\sigma$ are inapplicable and must be replaced by internal boundary conditions. We assume that these conditions consist of the continuity of $\Phi$ (dynamic condition) and the normal wind component (kinematic condition) across the boundary. Thus for a vertical internal boundary $(\sigma$ discontinuous horizontally)

$$
\left.\begin{array}{rl}
v_{n}^{(1)} & =v_{n}^{(2)} \\
\Phi^{(1)} & =\Phi^{(2)}
\end{array}\right\}
$$

where $v_{n}$ is the wind component normal to the boundary, or for a horizontal boundary

$$
\left.\begin{array}{l}
\omega^{(1)}=\omega^{(2)} \\
\Phi^{(1)}=\Phi^{(2)}
\end{array}\right\}
$$

The superscripts (1) and (2) refer to opposite sides of the boundary.

Since $\bar{\alpha}$, and therefore $\sigma$, is not a function of time, a separable time solution may be assumed, consisting of positive and negative exponent terms. We are interested in unstable waves, and we therefore ignore the negative exponential and write,

$$
\omega=e^{q t} \Omega(x, y, p)
$$

where $\Omega$ is the spatial function satisfying the reduced differential equation

$$
\nabla^{2}(\sigma \Omega)+\left(f^{2}+q^{2}\right) \frac{\partial^{2} \Omega}{\partial p^{2}}=0 .
$$

Inspection of table 1 shows that, as is generally known, static stability is negative with respect to the moist adiabatic process in the low levels of the tropical troposphere in summer, but is positive for dry motions. Since the atmosphere in these regions usually contains substantial amounts of moisture it is frequently only a small exaggeration to say that all upward motion proceeds at the unstable moist adiabatic lapse rate and all downward motion at the stable dry rate. If then, we equate saturated cloudy regions with negative values of $\sigma$ (and $\omega$ ) and dry clear regions with positive $\sigma$ (and $\omega$ ), equation (17) becomes a mixed elliptic-hyperbolic type equation with an internal boundary condition. The general theory of such systems is far from well established, although they are frequently encountered in the study of transonic flow (see, e.g., Frankl [5]). No method has been devised for obtaining an analytic or numerical solution of the system with realistic values of $\sigma$ in both the moist and dry regions. It is relatively simple, however, to obtain solutions for $\sigma$ constant or a variable function of only one coordinate. In this way we may qualitatively approach the results of a solution of the physically realistic system by considering three general types of solution, corresponding to three approximations to the true stability function.

Type I solutions are obtained for $\sigma$ equal to a constant. The system here is similar to that used by Syono and yields results, in particular the dynamic stability criterion, which set a standard of comparison for more complicated cases. For type II we allow $\sigma$ to vary in the horizontal only, being negative in moist regions and positive elsewhere, and we further restrict it and all other quantities to one-dimensional variation. Type III solutions are obtained under the assumption that $\sigma$ is constant horizontally but variable in the vertical. This allows consideration of the effects of stable layers and the tropopause.

It is possible to obtain simple solutions for a fourth type of stability variation; that is, where $\sigma$ is a function of pressure multiplied by a function of horizontal space. Such a solution was considered by Haque, but the resúlts are not particularly meaningful. Table 1 shows that $\sigma_{w}$ and $\sigma_{d}$ are of approximately equal magnitudes and opposite signs in the low levels, but are essentially identical above $200 \mathrm{mb}$. Thus this fourth type of solution is not a substitute for the presently unobtainable solution for the realistic form of $\sigma$.

\section{TYPE I SOLUTIONS}

For $\sigma$ equal to a constant, one can immediately separate variables in (17) and obtain the reduced differential equations in the form

$$
\begin{gathered}
\frac{d^{2} P}{d p^{2}}+\frac{K^{2}}{p_{0}{ }^{2}} P=0 \\
\nabla^{2}(\sigma W)-\frac{f^{2}+q^{2}}{p_{0}^{2}} K^{2} W=0
\end{gathered}
$$

where $\Omega=P(p) \quad W(x, y)$ and $\left(K / \mathrm{p}_{0}\right)^{2}$ is the separation constant. If boundary conditions (8) and (12) are applied to (18), it is found that $K$ is an eigenvalue such that

$$
K=n \pi, \quad n=1,2, \ldots
$$

The use of boundary conditions (8) and (11) leads to a somewhat more complicated relation 


$$
K \tan K=-\sigma p_{0} / \bar{\alpha}_{0} .
$$

The right-hand side is much smaller than unity, and solutions of (21) may be found by expansion of the left side about the approximate (zero-order) solutions given by (20). To the first order these solutions are

$$
K= \begin{cases}\left(-\sigma p_{0} / \bar{\alpha}_{0}\right)^{1 / 2}, & n=0 \\ n \pi-\frac{\sigma p_{0}}{n \pi \bar{\alpha}_{0}}, & n=1,2, \ldots\end{cases}
$$

For $n \geq 1$ the eigenvalues obtained from the more accurate lower boundary condition are essentially unchanged from those of the more approximate case. The solution for $n=0$ represents an external gravity wave which is not of interest in this study. We are therefore justified in using lower boundary condition (12) as sufficiently accurate for the present purposes.

Elementary solutions to (19) may be found in any of several forms, depending on the coordinate system used. For simplicity we choose to specialize the system to one-dimensional variation in either the Cartesian or cylindrical coordinate system with symmetry about the origin and $W$ always finite. Thus $W$ is either an even function of $x$ or a function of $r$. In either case the coefficient of the second term of (19) must also assume discrete values (eigenvalues) and we may write

$$
\nabla^{2} W+(k / L)^{2} W=0
$$

where $k=\pi / 2$ in the Cartesian case, $k \approx 2.405$ (the first zero of the Bessel function of order zero) in the cylindrical case, and $L$ is the distance from the origin to the point where $W$ first changes sign. The solutions for $\omega$ in the Cartesian case are as follows:

$$
\omega=A e^{q t} \sin \frac{n \pi p}{p_{0}} \cos \frac{k x}{L}, \quad k=\pi / 2 .
$$

The wave equation may be found as a condition which simultaneously satisfies (19), (20), and (23), that is

$$
q^{2}+f^{2}=-\left(\frac{p_{0} / n \pi}{L / k}\right)^{2} \sigma
$$

Solutions for $\Phi, \vee$, and $\alpha$ for the Cartesian case are as follows:

$$
\begin{gathered}
\Phi=\frac{p_{0} \sigma}{n \pi q} A e^{q t} \cos \frac{n \pi p}{p_{0}} \cos \frac{k x}{L} \\
\mathrm{~V}=\frac{k p_{0}}{n \pi L} \frac{\sigma(q \mathbf{i}-f \mathbf{i})}{q\left(q^{2}+f^{2}\right)} A e^{q t} \cos \frac{n \pi p}{p_{0}} \sin \frac{k x}{L} \\
\alpha=(\sigma / q) \omega .
\end{gathered}
$$

Circularly symmetric solutions are identical to the above except for the replacement of cosine and sine functions by Bessel functions of order zero and one, respectively.
Figure 1 shows horizontal profiles of the above solutions for $\omega, \Phi$, and the divergent velocity component, and figure 2 illustrates the vertical variation of $\omega$ and $\mathcal{D}$, the horizontal divergence. We see that for real positive $q$, upward motion in the center $(A<0)$ corresponds to negative perturbation geopotential and convergent cyclonic indraft in the lower levels $\left(p / p_{0}>0.5\right)$ and to positive geopotential and anticyclonic divergent outdraft in the upper levels. The specific volume (and temperature) perturbation has its maximum in the middle levels, and is positive for $\sigma<0$. The ratio of the divergent to the rotational wind velocity can be seen, from (27), to be equal to $q / f$, which the wave equation shows to be a function of static stability and the scale of motion. We also note that solutions for all the dependent variables oscillate out to infinity. This will not be true for subsequent cases in which $\sigma$ is allowed to vary with the sign of the vertical motion.

The dynamic stability criterion arising from wave equation (25) is the following:

$$
\left(\frac{L}{p_{0} / n}\right)^{2} \leq-\sigma\left(\frac{k}{\pi f}\right)^{2}\left\{\begin{array}{l}
\text { Unstable } \\
\text { Neutral } \\
\text { Stable }
\end{array}\right.
$$

Unstable solutions may exist only for $\sigma<0$ and then only if the ratio of the horizontal to the vertical scale is sufficiently small. For example, if we set in $\sigma=-3 \times 10^{-3}$ m. ${ }^{2} \mathrm{mb}^{-2}$ sec. $^{-2}$ as a reasonable order of magnitude (justified in a later section) we find that unstable waves may exist at latitude $20^{\circ}$ for $L<500 \mathrm{~km}$. approximately, ' or roughly the size range of large tropical cyclones. If the stability criterion indicates imaginary $q$, then the solutions (24)-(28) represent standing inertio-gravitational waves. Traveling wave solutions are also possible, of course, and such waves will have a wave speed, in the Cartesian system, equal to $2|q| L / \pi$.

Equation (25) indicates that $q$ approaches infinity if $L / p_{0} \rightarrow 0$. This obviously unreasonable result is due to the quasi-static assumption and neglect of acceleration terms in the vertical equation of motion. If these terms are included in a derivation in the $x, y, z$ system, as was exhibited by Haque [8], and the terms in the resulting wave equation transformed to a similar form to those in (25), the latter is modified as follows:

$$
q^{2}=\frac{-f^{2}-\sigma\left(\frac{p_{0} / n \pi}{L / k}\right)^{2}}{1+\left(\frac{\bar{\alpha}}{g} \frac{p_{0} / n \pi}{L / k}\right)^{2}}
$$

where $\overline{\bar{\alpha}}$ is a specific volume averaged through the depth of the system. Evaluation of the second term of the denominator shows it to be small for $L \gg 10 \mathrm{~km}$., i.e. (30) reduces to (25), but rapidly increasing for smaller disturbances. This corresponds roughly to the scale of motion for which nonhydrostatic terms are of impor- 

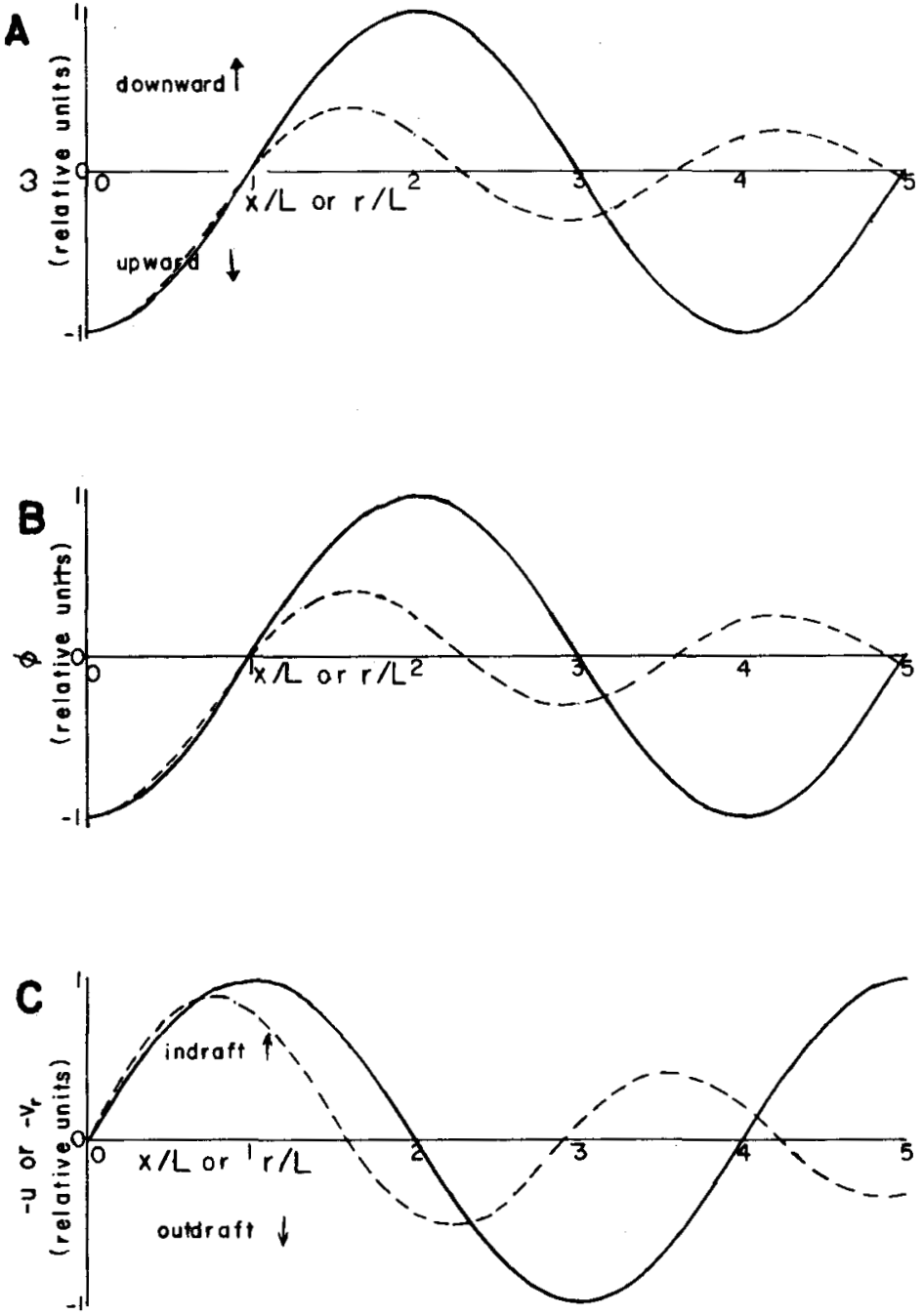

Figure 1.-Type I horizontal solutions, moist motions everywhere. Slab symmetry (solid), eircular symmetry (dashed). (A) $\omega$ at $p=p_{0} / 2$. (B) $\Phi$ at $p=p_{0}$. (C) $(-u)$ or $\left(-v_{r}\right)$ at $p=p_{0}$.

tance. As $L / p \rightarrow 0$, that is, for very narrow, "tall" disturbances, (30) reduces to

$$
q^{2}=-\left(\frac{g}{\overline{\bar{\alpha}}}\right)^{2} \sigma
$$

which then represents a finite maximum growth rate and, of course, indicates that the Coriolis term is unimportant for small scales of motion. The dependence of $q$ on horizontal scale and static stability is shown in figure 3 , in which $n=1, p_{0}=1000 \mathrm{mb} ., \overline{\bar{\alpha}}=1.4 \times 10^{3} \mathrm{~cm} .^{3} \mathrm{gm} \cdot{ }^{-1}$, $\boldsymbol{k}=\pi / 2$, and $f=5 \times 10^{-5} \mathrm{sec}^{-1}$

Equation (30) and figure 3 show that the maximum growth rate occurs for $L=0$. Since observations show that most rapidly growing cloud convection cells are roughly as wide as they are tall, one might expect to find the real maximum growth rate to exist for $L \approx 10 \mathrm{~km}$. This discrepancy is caused by the neglect of internal

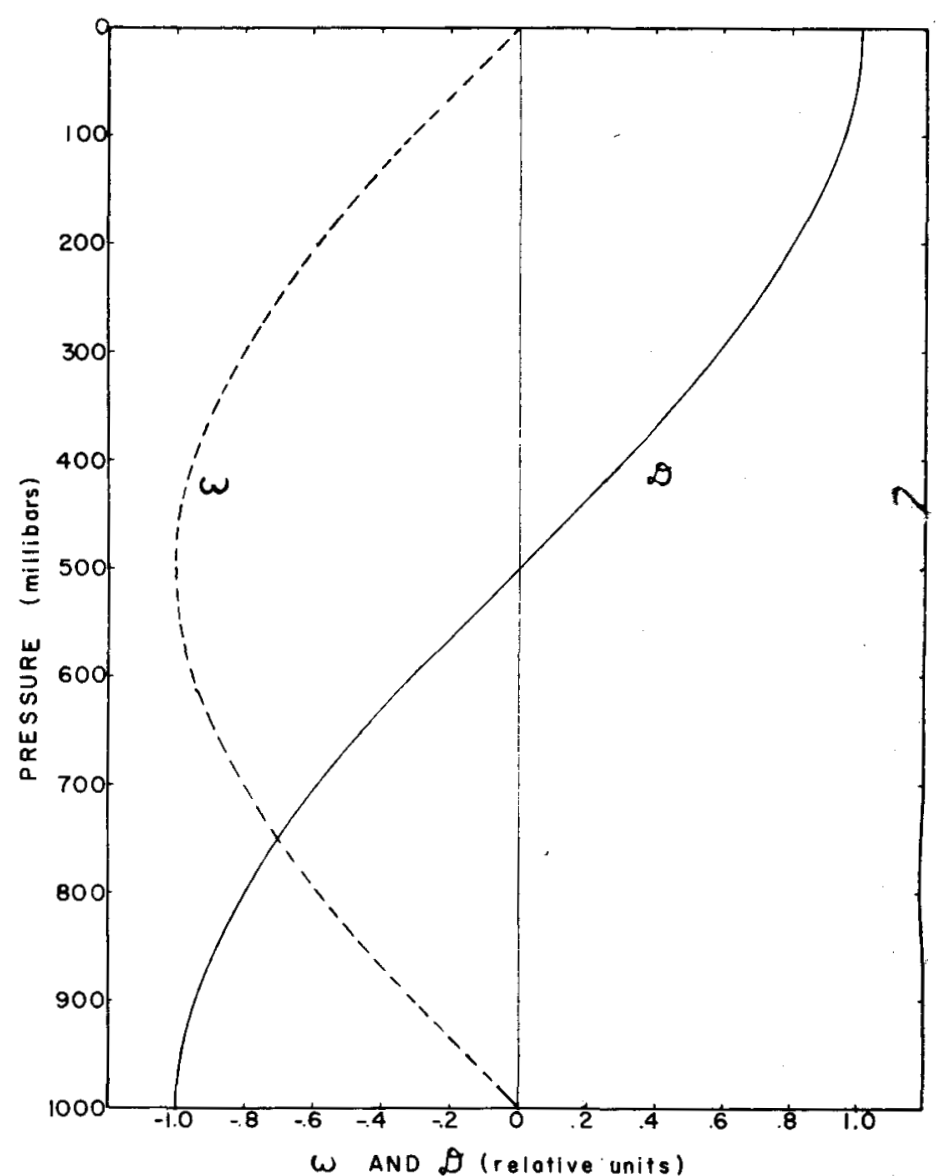

Figure 2.-Type I vertical solutions. $\mathcal{D}$ (soid line), $\omega$ (dashed line) scale units normalized by maximum abscissae.

viscosity, diffusion, and entrainment effects in the present model (cf. Godske et al. [7], chapter 9).

A more important discrepancy between the stability theory and observations lies in the frequent occurrence of large-amplitude convective disturbances of horizontal dimensions much greater than those having the maximum linear growth rate. Full understanding of this bimodal tendency will doubtless require thorough consideration of nonlinear and dissipative effects, and the role of largescale, quasi-steady-state motions in accumulating moisture at higher altitudes than normal. Linear theory can provide certain necessary conditions for further development of preexisting disturbances of a given scale and shape. It can say a little about the expected relative frequency of various disturbance scales, and also say a little about the effects of widely different scales upon each other.

\section{TYPE II SOLUTIONS}

We next consider the effects of the horizontal variation of static stability and, in particular, the variation caused by a discontinuous change in the polytropic exponent, as 


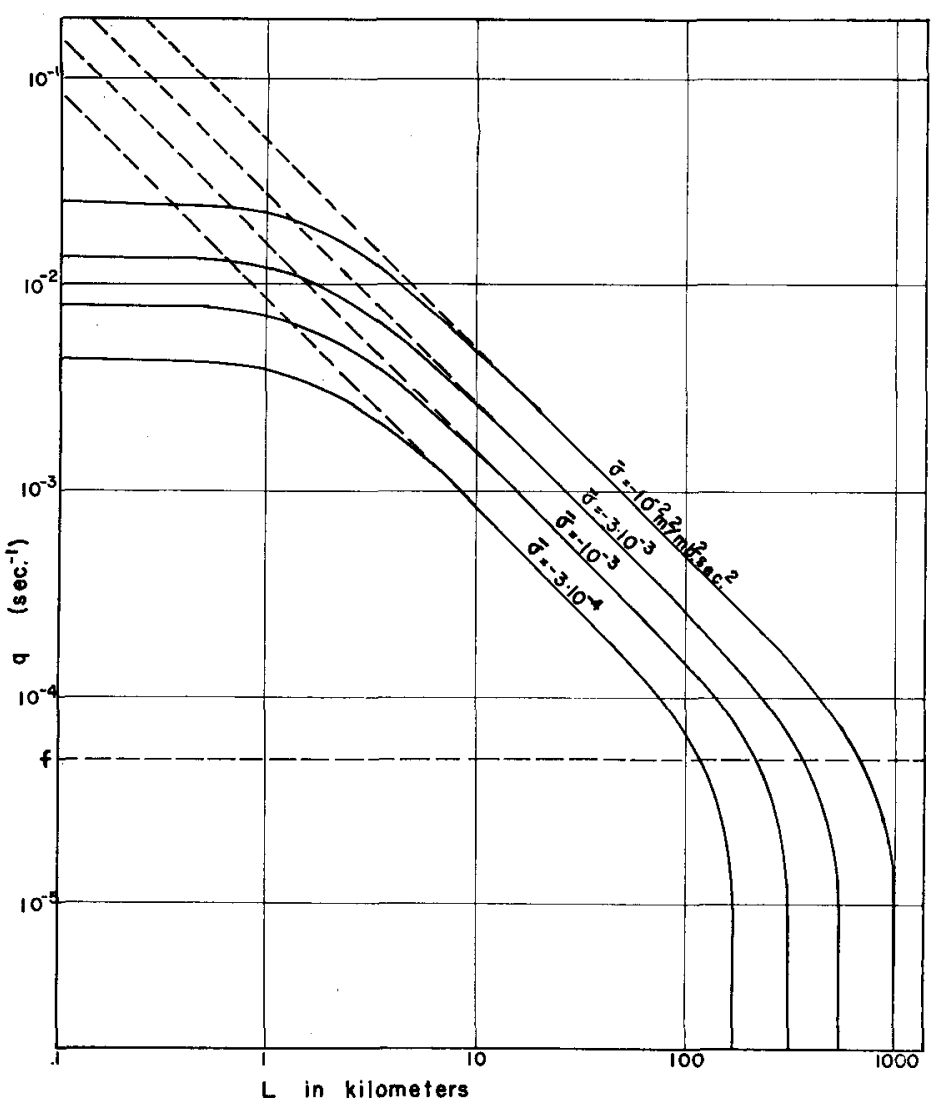

Figure 3.--Wave equation (25), dotted line, and (30), solid line, * for various values of $\sigma$ shown on the curves. $f=5 \times 10^{-5}$ sec. $^{-1}$, $p_{0}=1000 \mathrm{mb} . \quad n=1, k=\pi / 2$.

from a moist to a dry process. We assume for simplicity that $\sigma$ is a negative constant in one or more moist regions and a positive constant elsewhere. Of the various possible geometric arrangements of the moist and dry regions, the following cases have been chosen for presentation as holding particular physical interest.

Case 1: Moist (statically unstable) ascent in the center, surrounded by dry (statically stable) descent out to infinity:

(a) Slab symmetry (Cartesian coordinates).

(b) Circular symmetry (cylindrical coordinates).

Case 2: Same as case 1 except horizontally bounded by rigid walls at finite distances (slab symmetry only).

Case 3: Dry descent in the center, surrounded by moist ascent and dry descent outside to infinity (slab).

Case 4: Moist ascent in the center, surrounded by (infinitely) many alternating bands of dry descent and moist ascent, dry descent outside to infinity (slab).

\section{CASE 1}

Case 1 is the simplest possible model in which compensating dry descent is included. The principal result of the development is the appearance of a transcendental equation obtained from application of the internal boundary condition to the otherwise independent solutions for the moist and dry regions. This transcendental, which we call the compatibility condition, serves to define a dynamically effective static stability, $\bar{\sigma}$, in terms of the values of $\sigma$ in the moist and dry regions and their geometric relationships. We then can replace $\sigma$ by $\bar{\sigma}$ in the wave equations and stability criteria derived for the type I solution and the latter maintain their essential significance. Also in case 1 the differences between circular and slab symmetry are investigated and presented.

We consider two solutions to equations (17), (8), and (12), one valid within an inner region, $|x|$ or $r<L$, where $\sigma=\sigma_{w}<0$, and the second outside this region, where $\sigma=\sigma_{d}>0$. Thus $L$ is now defined as the half-width or radius of the moist motions only. For each of the regions variables may be separated in (17) exactly as for type I, and solutions to equation (18) are identical with those of the former example. We will henceforth in this section set $n=1$; i.e., only one vertical mode of oscillation will be considered. Finally we define $\bar{\sigma}$ to satisfy the wave equation, that is

$$
q^{2}+f^{2}=-\bar{\sigma}\left(\frac{p_{0} / n \pi}{L / k}\right)^{2}
$$

Under these conditions the horizontal differential equation (19) may be solved separately for the moist and dry regions with apparently independent amplitude constants. Application of the internal boundary conditions (14), however, constrains these constants and further generates a condition of compatibility between the solutions for the adjoining regions, which is for the slab and circular symmetry case respectively.

$$
\tan \left[k\left(\bar{\sigma} / \sigma_{w}\right)^{1 / 2}\right]=\left(-\sigma_{w} / \sigma_{d}\right)^{1 / 2}, k=\pi / 2
$$

$\frac{J_{1}\left[k\left(\bar{\sigma} / \sigma_{w}\right)^{1 / 2}\right] K_{0}\left[k\left(-\bar{\sigma} / \sigma_{d}\right)^{1 / 2}\right]}{J_{0}\left[k\left(\bar{\sigma} / \sigma_{w}\right)^{1 / 2}\right] K_{1}\left[k\left(-\bar{\sigma} / \sigma_{d}\right)^{1 / 2}\right]}=\left(\frac{-\sigma_{w}}{\sigma_{d}}\right)^{1 / 2}, k=2.405 \ldots$

where $J_{n}$ and $K_{n}$ are the $n$ th-order Bessel functions of real and imaginary argument, respectively, which vanish at infinity (see Watson [23]).

The solutions for $\omega$ in the moist and dry regions for slab symmetry are as follows:

$$
\omega_{w}=A e^{a t} \sin \frac{\pi p}{p_{0}} \cos \frac{k\left(\bar{\sigma} / \sigma_{w}\right)^{1 / 2} x}{L},|x|<L
$$

$$
\begin{aligned}
\omega_{d}=\frac{\sigma_{w t}}{\sigma_{d}} \cos \left[k\left(\bar{\sigma} / \sigma_{w}\right)^{1 / 2}\right] A e^{q t} \sin \frac{\pi p}{p_{0}} & \\
& \times \exp \left[k\left(-\bar{\sigma} / \sigma_{d}\right)^{1 / 2}(1-|x| / L)\right],|x|>L
\end{aligned}
$$

Solutions for the remaining independent variables and the circularly symmetric solutions are related to those above in a similar manner with (24)-(28).

The horizontally variable part of the solutions for $\omega$, $\Phi$, and $u$ or $v_{r}$ (radial velocity) are shown in figure 4 . In comparing these with type I we note, first, the difference in behavior for large $|x|$ or $r$. In this case the geopotential, vertical and horizontal velocity, etc., fall off exponentially 

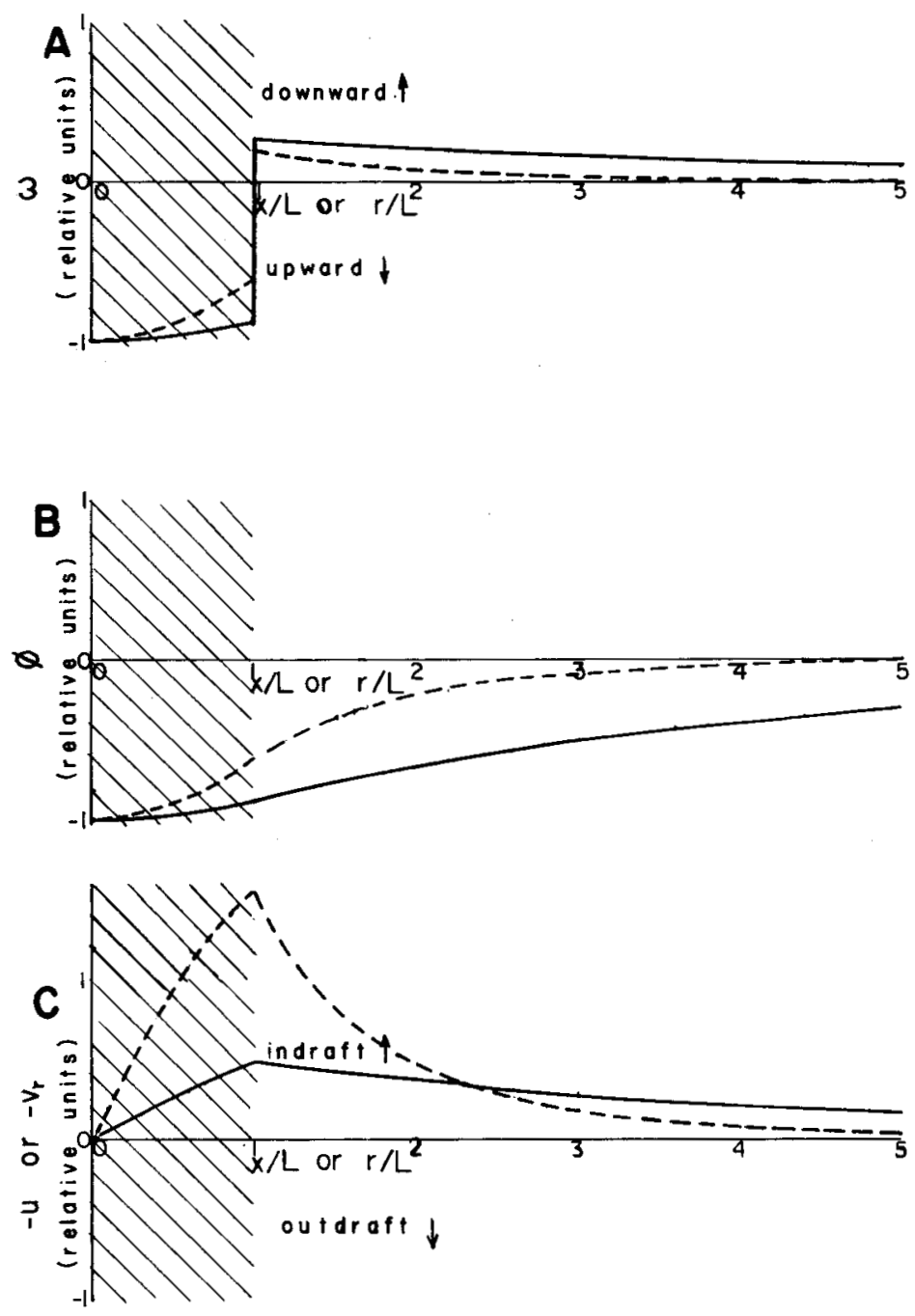

Figure 4.-Type II, case 1, horizontal solutions, moist region hatched. Slab symmetry (solid), circular symmetry (dashed). (A) $\omega$ at $p=p_{0} / 2$. (B) $\Phi$ at $p=p_{0}$. (C) $(-u)$ or $\left(-v_{r}\right)$ at $p=p_{j}$.

in the dry region, showing the passive forced nature of the dry motions. At the same time the dry motions act somewhat as a brake upon the instability. The ratio of the effective stability to the moist stability, $\bar{\sigma} / \sigma_{w}$, has been computed for both the slab and circular solutions for various values of $\sigma_{w} / \sigma_{d}$, and is shown in table 2 .

We note that the reduction is much greater for slab than for circular symmetry, thus favoring development of the latter. The reason for this behavior seems to lie in the more compact nature of the circular disturbance.

TABLE 2.-The ratio of effective stability to moist stability for various values of the ratio of moist to dry stability

\begin{tabular}{l|c|c|c|c|c|c|c}
\hline \hline$-\sigma_{u} / \sigma_{d-\ldots}$ & 0.0 & 0.1 & 0.3 & 0.5 & 1.0 & 10.0 & $\infty$ \\
\hline$\tilde{\sigma} / \sigma_{w}$ Circular $\ldots$ & 0.0 & 0.211 & 0.299 & 0.357 & 0.451 & 0.760 & 1.000 \\
\hline$\sigma / \sigma_{w}$ Slab_............. & 0.0 & 0.037 & 0.101 & 0.153 & 0.250 & 0.624 & 1.000 \\
\hline
\end{tabular}

The plotted curves for $\Phi, \omega$, and $V$ show that these functions have their largest amplitudes within and very close to the moist region for the circular case, but exhibit a much slower damping in the case of slab symmetry. Thus the latter may be effectively "larger" and hence less active than the former, for a given width of the moist region. Thus bands seem to be less efficient than circular disturbances for release of convective instability and the large frequency of occurrence of such bands must be explained as a consequence of the vertical wind shear or other effects, e.g., turbulent dissipation, not considered in this study.

The solutions for $V$ show that the maximum radial and tangential winds both occur at the internal boundary, that is, at the outer limit of the moist region. In section 9 it is shown that nonlinear terms have a considerable effect on the tangential wind distribution at rather small disturbance amplitudes. It may be noted that $\omega$, horizontal divergence, and relative vorticity (not shown) are discontinuous at the internal boundaries. In the real atmosphere these discontinuities would presumably be replaced by sharp gradients. In the dry region relative vorticity is negative, corresponding to conditions in the outskirts of tropical cyclones as observed by several Japanese meteorologists (Ootani and Hatakeyama [16]; Syōno et al. [22]).

\section{CASE 2}

The boundary conditions for case 2 are identical to those obtainable under the assumption that another disturbance of identical scale and amplitude adjoins on either side of the one under consideration. Thus we may investigate the behavior of a convective band and the constraints imposed upon it by the existence of neignboring bands. An interesting result is the appearance of a limitation on the ratio of horizontal extent of the moist and dry regions for existance of unstable solutions; that is, a limiting proximity of adjoining bands. The external boundary condition here consists of vanishing of the wind component normal to the wall. After applying this condition to the elementary solutions of (17), it is found that $\omega_{w 0}$ is unchanged, but $\omega_{d}$ is replaced, for the slab symmetric case, by an expression involving hyperbolic cosines. The fields of $\omega, \Phi$, and $u$ are illustrated, for this case, in figure 5. The compatibility condition is altered to the following form:

$$
\frac{\tan \left[k\left(\bar{\sigma} / \sigma_{w}\right)^{1 / 2}\right]}{\tanh \left[k\left(-\bar{\sigma} / \sigma_{d}\right)^{1 / 2}\left(x_{1}-L\right)\right]}=\left(-\frac{\sigma_{w}}{\sigma_{d}}\right)^{1 / 2}
$$

where $\pm x_{1}$ is the abscissa of the external boundary, and thus $x_{1}-L$ is the half-width of the dry region.

Examination of (36) shows that the horizontal constraint reduces the effective instability, and $\bar{\sigma}$ vanishes for $\left(x_{1}-L\right) /$ $L=-\sigma_{d} / \sigma_{w}$. This is the minimum ratio of the separation of convective bands to their width, i.e. the clear-cloud ratio, for which the bands may amplify. Depending on the vertical dimension of the disturbances, table 1 indicates that this ratio may vary from about one to three in the mean tropical troposphere. This revision of the stability 

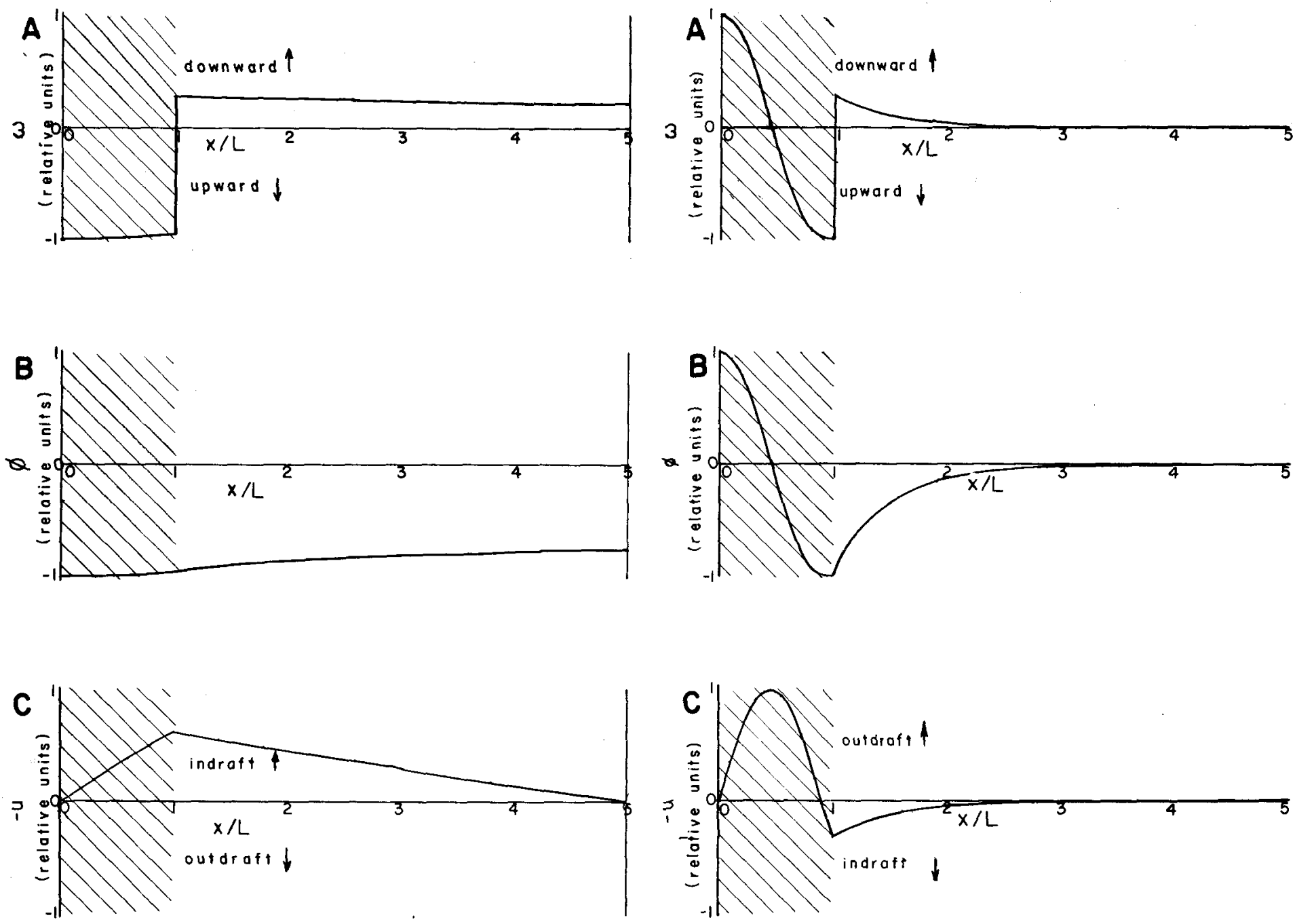

Figure 5.-Type II, case 2, horizontal solutions, moist region hatched. (A) $\omega$ at $p=p_{0} / 2$. (B) $\Phi$ at $p=p_{0}$. (C) $(-u)$ at $p=p_{0}$.

criterion bears a close relation to the conclusions of the "slice" method of forecasting convection, first introduced by J. Bjerknes [2].

\section{CASE 3}

Taking the radius of the "eye" equal to $a$, where $a<L$, we specify a stability distribution as follows:

$$
\left.\begin{array}{ll}
\sigma=\sigma_{d}, & |x|<a \\
\sigma=\sigma_{w}, & a<|x|<L \\
\sigma=\sigma_{d}, & |x|>L .
\end{array}\right\}
$$

The horizontal solution for $\omega$, with slab symmetry, is proportional to the hyperbolic cosine in the eye, sinusoidal in the moist region, and as before, a decreasing exponential outside. The horizontal profiles of $\omega, \Phi$, and $u$ are exhibited in figure 6 ; circularly symmetric solutions are similar in appearance. The compatibility condition becomes

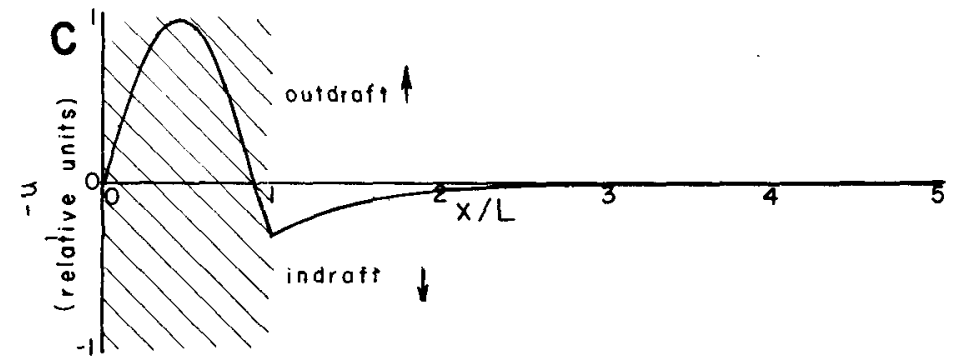

Figure 6.-Type II, case 3 , horizontal solutions, moist region hatched. (A) $\omega$ at $p=p_{0} / 2$. (B) $\Phi$ at $p=p_{0}$. (C) $(-u)$ at $p=p_{0}$.

$$
\begin{aligned}
& \tan \left[\frac{k\left(\bar{\sigma} / \sigma_{w}\right)^{1 / 2}(L-a)}{L}\right] \\
& =\left(\frac{-\sigma_{w}}{\sigma_{d}}\right)^{1 / 2} \frac{1+\tanh \left[k\left(-\bar{\sigma} / \sigma_{d}\right)^{1 / 2} a / L\right]}{1+\left(\sigma_{w} / \sigma_{d}\right) \tanh \left[k\left(-\bar{\sigma} / \sigma_{d}\right)^{1 / 2} a / L\right]}
\end{aligned}
$$

By comparison of (38) with (33), it can be seen that $\bar{\sigma}$ is greater for this case, and the "eye" tends to increase the grow th rate. The amount of this increase however, is insignificant. For $\sigma_{w}=-0.3 \times 10^{-3}$ and circular symmetry, an eye with a radius of $0.1 L$ (1/10 that of the moist region) increases effective static instability by less than 2 percent, thus changing the wave equation only very slightly. Although the eye plays an indispensable part in maintenance of a tropical cyclone, as first shown by Haurwitz [9], it evidently does so by means of nonlinear terms not considered in this study.

The vertical motion distribution in the eye case, as shown in figure 6 , exhibits a downdraft region in the eye, 
associated with an indetectably slight surface high pressure area and slight-anticyclonic outdraft winds. The vorticity and vertical motion are again discontinuous at both internal boundaries.

\section{CASE 4}

This case was also picked to represent a certain physical feature of the tropical cyclone; that is, the observed existence of convective bands in the outer regions of the storm. Here we consider the integrated effect of a large number of smaller disturbances of the type considered in case 2 . A rather interesting conclusion of this case, in combination with the results of case 2 , is that a forcing disturbance is a necessary condition for development of large-scale unstable convective motion in the presence of smaller scale convection. This conclusion is a consequence of the effects upon a large-scale system of the dry descent surrounding smaller cells.

For this model it is assumed that an inner region of a developing cyclone-scale disturbance consists of many alternate bands of moist ascent and dry descent. It should be remembered that the growth exponent $q$ is assumed constant throughout, so that only the quasi-steadystate features of the meso-scale bands are considered. The shorter lived cloud-scale cells may be thought of as generating the proper conditions (moisture, especially) to assure maintenance of the bands and, by their compensating downdrafts and drying, to maintain spacing between the bands, according to the spacing requirements derived in case 2. A band is, for simplicity, considered at a constant distance from the disturbance center, rather than parallel to the low-level wind flow, as seems to be the actual case in tropical cyclones.

Methods used for finding solutions for the previous cases become tedious when a large number of narrow bands is assumed. Instead it is desirable to define some sort of averaged stability value appropriate to an area containing an infinite number of alternating moist and dry bands, which we do in the following manner. Consider two infinitesimal bands, one moist with stability $\sigma_{w}<0$ and the other dry with stability $\sigma_{d}>0$, and let the ratio of the width of the moist band to that of the total width of both be $U$. Since the bands are considered infinitesimal relative to the large-scale disturbance, we may consider that in each of the moist and dry bands $\Phi$ is approximately horizontally constant, and by application of (14) $\Phi$ is therefore approximately constant over both bands. Further, $\partial^{2} \Phi / \partial p \partial t$ is also approximately constant and, by application of (2). and (4), $\sigma_{w} \omega_{w} \approx \sigma_{d} \omega_{d}$, where $\omega_{w}$ and $\omega_{d}$ are the (approximately horizontally constant) vertical velocities in the moist and dry bands, respectively. If we define the average value of $\omega$ (across both bands) to be $\bar{\omega}=U \omega_{w}+(1-U) \omega_{d}$, then $\tilde{\sigma} \bar{\omega}=\sigma_{w} \omega_{v}=\sigma_{d} \omega_{d}$ if

$$
\frac{1}{\tilde{\sigma}}=\frac{U}{\sigma_{n}}+\frac{1-U}{\sigma_{d}}
$$

Thus $\tilde{\sigma}$ relates the average expansion of air to the average vertical motion and could be alternatively defined as

$$
\tilde{\sigma}=\frac{\iint \sigma \omega d x d y}{\iint \omega d x d y}
$$

where the integral is taken over the banded region. If $U$ is constant, (39) and (40) can be shown to be equivalent. We define $\tilde{\sigma}$ to be the band stability.

From (40) one can see the possibility of an infinite value of $\tilde{\sigma}$, if $U=-\sigma_{w} /\left(\sigma_{d}-\sigma_{w}\right)$, implying that heat is released from vertical motions, but the mean vertical motion is zero. In this case divergence and relative vorticity vanish. Hughes [11] has shown this to be approximately the condition in the outer rain bands of tropical cyclones.

An even more significant conclusion comes from a comparison of relation (39) with the conclusions of case 2 . In case 2 we look at the convective band as a meso-scale phenomenon, associated with disturbances of small size and rather rapid time variations, while in case 4 we consider the band from the large-scale quasi-steady-state point of view, disregarding all transient features. In equation (36) and subsequent discussion it was shown that a small-scale disturbance could grow only if the ratio of moist to dry area is less than or equal to the ratio of dry to moist stability. On the other hand, (39) indicates that the band stability for large-scale disturbances is negative only if the ratio of moist to dry area is greater than the ratio of dry to moist stability. This means that a cyclonescale convective disturbance cannot develop as simply an amalgamation of smaller-scale cells, but must include at least a meso-scale central core of moist ascent, presumably generated by a disturbance whose energy is derived from other than convective instabilities.

Having postulated the existence of such a core we now assume three distinct regions with different values of stability; i.e.,

$$
\left.\begin{array}{l}
\sigma=\sigma_{u}<0, \quad|x|<b \\
\sigma=\sigma_{b} \rightarrow-\infty, \quad b<|x|<2 L-b \\
\sigma=\sigma_{d}>0, \quad|x|>2 L-b
\end{array}\right\}
$$

where $2(L-b)$ is the width of the banded region. The solutions for $\Phi$ in the inner and outer regions are similar to those of case 1, while that for the banded region shows a linear variation with $x$ (logarithmic with $r$ for circular symmetry). These solutions are exhibited in figure 7 . The compatibility condition is found to be the following:

$$
\tan \left[k\left(\bar{\sigma} / \sigma_{w}\right)^{1 / 2} b / L\right]=\left[\left(-\sigma_{d} / \sigma_{w}\right)^{1 / 2}-2 k\left(\bar{\sigma} / \sigma_{w}\right)^{1 / 2}(L-b) / L\right]^{-1}
$$

which shows that the banded region has a dynamic effect midway between that of an all-dry and an all-moist region. 


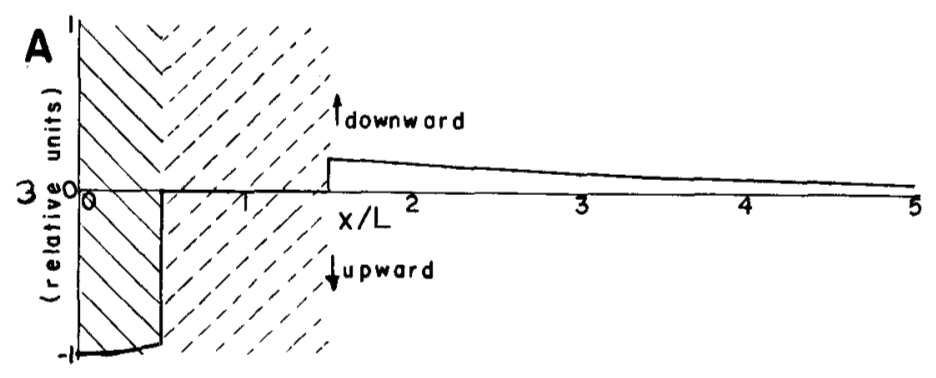

pressures above $100 \mathrm{mb}$, constant temperature for pressures below $100 \mathrm{mb}$.

\section{CASE 1}

Here we investigate the effects of a sharp tropopause discontinuity in static stability. It is shown that the stratosphere acts effectively as a lid to the disturbance and can be considered as equivalent to applying a solid surface boundary condition at a level slightly above the tropopause.

We prescribe $\sigma$ for the lower and upper layers, respectively, by the following expressions:

$$
\begin{gathered}
\sigma=\sigma_{\mathbf{T}}<0 \text { for } p>p_{\mathrm{T}} \\
\dot{\sigma}=\sigma_{\mathrm{S}}=R^{2} \bar{T}_{\mathrm{S}} / c_{p} p^{2} \text { for } p<p_{\mathbf{T}}
\end{gathered}
$$

where $\sigma_{\mathrm{T}}$ is a negative constant and equation (44b) is obtained from (7) with $\bar{T}_{\mathrm{s}}$ the stratosphere temperature. Solutions to (18), with $K$ evaluated from (43), (44a), and $(44 \mathrm{~b})$, satisfying the external boundary conditions (8) and (12) may be written as follows:

$$
\begin{gathered}
P=A_{\mathrm{T}} \sin \left[\pi\left(\sigma_{\mathrm{T}} / \sigma\right)^{1 / 2}\left(p_{0}-p\right) / p_{0}\right], p>p_{\mathrm{T}} \\
P=A_{\mathrm{S}}\left(p / p_{0}\right)^{1 / 2+N}, p<p_{\mathrm{T}}
\end{gathered}
$$

where

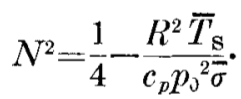

Internal boundary conditions (15) lead to a relation between the amplitude coefficients $A_{\mathrm{s}}$ and $A_{\mathrm{r}}$ and to a compatibility condition which can be solved to yield $\bar{\sigma}$. This may be written

$$
\tan \left[\pi\left(\sigma_{\mathbf{T}} / \bar{\sigma}\right)^{1 / 2}\left(p_{0}-p_{\mathbf{T}}\right) / p_{0}\right]=-\left(N+\frac{1}{2}\right)\left[\pi\left(\sigma_{\mathbf{T}} / \sigma\right)^{1 / 2} p_{\mathbf{T}} / p_{0}\right]
$$

Solution of (46) shows that $\bar{\sigma} / \sigma_{w}<1$. We now show that reduction of instability by the presence of the stratosphere is identical to the effect of a rigid boundary at $p=p_{\mathrm{H}}$, where $p_{\mathrm{H}}\left\langle p_{\mathrm{T}}\right.$. We set $\sigma=\sigma_{\mathrm{T}}$ for all $p>p_{\mathrm{H}}$, and define $p_{\mathbf{H}}$ by the relation

$$
\left(p_{0}-p_{\mathbf{H}}\right) / p_{0}=\left(\bar{\sigma} / \sigma_{\mathbf{T}}\right)^{1 / 2}
$$

Substitution of $(47)$ into (45a) shows that $P$, and therefore $\omega$, vanishes at $p=p_{\mathrm{H}}$. Values of $p_{\mathrm{H}}$ and $\bar{\sigma} / \sigma_{\mathrm{T}}$ computed from (46) and (47) for various values of $p_{\mathrm{T}}$ are given in table 3 .

TABLE 3.-The equivalent rigid tropopause level, $p_{\mathbf{B}}$, and the effective static stability, $\bar{\sigma}$, for various values of the tropopause pressure, $p_{\mathbf{T}}$

\begin{tabular}{c|r|r}
\hline \hline$p_{\mathbf{T}}(\mathrm{mb})$. & \multicolumn{1}{c|}{$\boldsymbol{\sigma} / \sigma_{\mathbf{T}}$} & \multicolumn{1}{|c}{$p_{\text {H }}(\mathrm{mb})}$. \\
\hline 100 & 0.914 & 86 \\
150 & .870 & 130 \\
200 & .826 & 174 \\
\hline
\end{tabular}




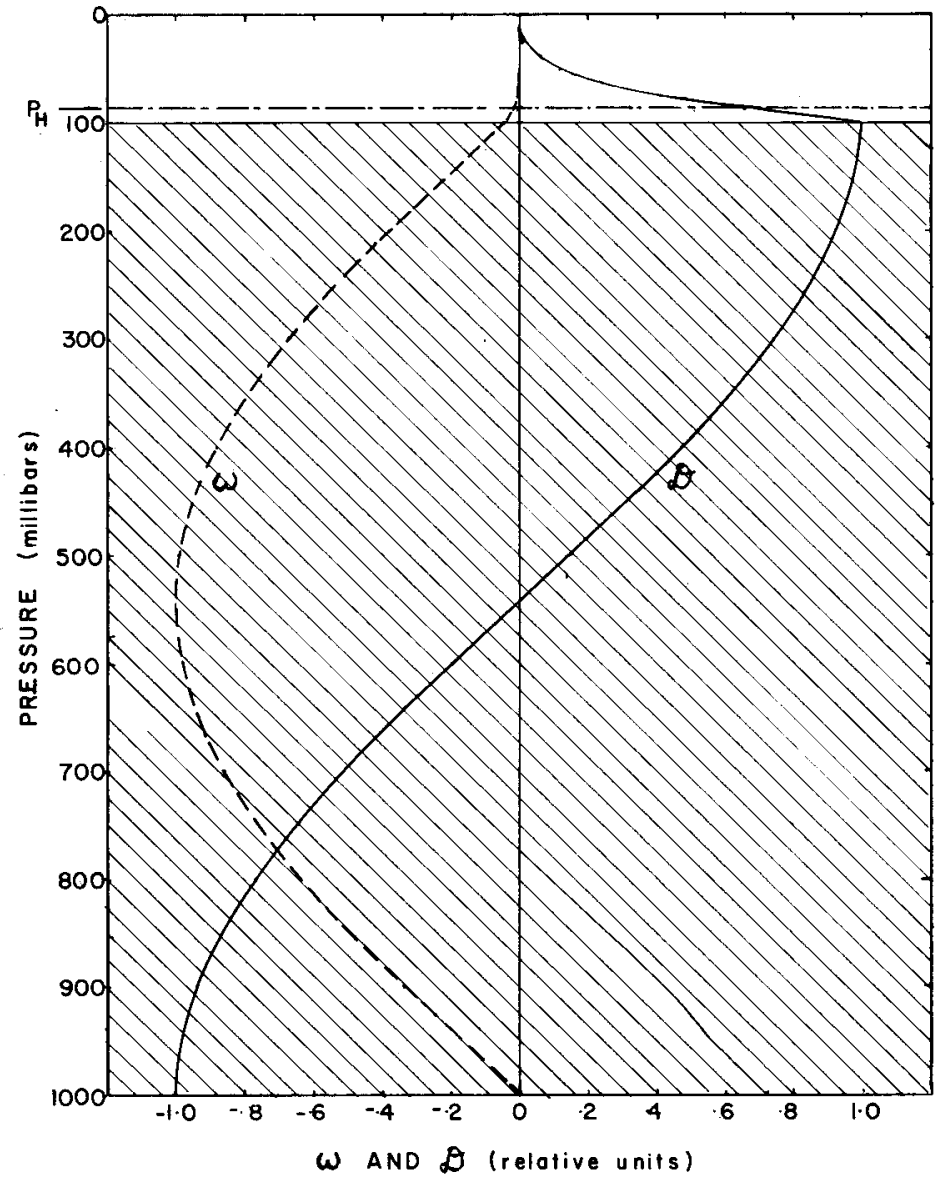

Figure 8.-Type III, case I, vertical solutions $\mathcal{D}$ (solid line), $\omega$ (dashed line), scale units normalized by maximum abscissae. Dash-dotted line represents equivalent rigid tropopause. Hatched area is statically unstable.

Solutions of case 1 for $\omega$ and $D$ are presented in figure 8 for $p_{\mathrm{T}}=100 \mathrm{mb} ., \quad \sigma_{\mathrm{T}}=-3 \times 10^{-3} \mathrm{~m} \cdot{ }^{2} \mathrm{mb} \cdot{ }^{-2} \mathrm{sec}{ }^{-2}$, and $\bar{T}_{\mathrm{s}}=200^{\circ} \mathrm{K}$. The curves further illustrate the extremely rapid decrease of disturbance amplitude above the tropopause.

\section{CASE 2}

Here the distribution of stability with height is relatively smooth. The results of the numerical integration of (18) for this case show, however, that again the principal effect of an upper stable layer is to diminish the effective depth of a disturbance and reduce its growth rate correspondingly.

The solution for this case was found by numerical integration of (18) using (43), and with $\sigma=\sigma_{w}$, evaluated from table 1 for $p>100 \mathrm{mb}$., and $\sigma$ taken from (44b) for $p<100 \mathrm{mb}$., where $\bar{T}_{\mathrm{s}}=200^{\circ} \mathrm{K}$. The Gill [6] formulation of the Runge-Kutta integration scheme, as programmed for the IBM 704, was utilized in forward integration from

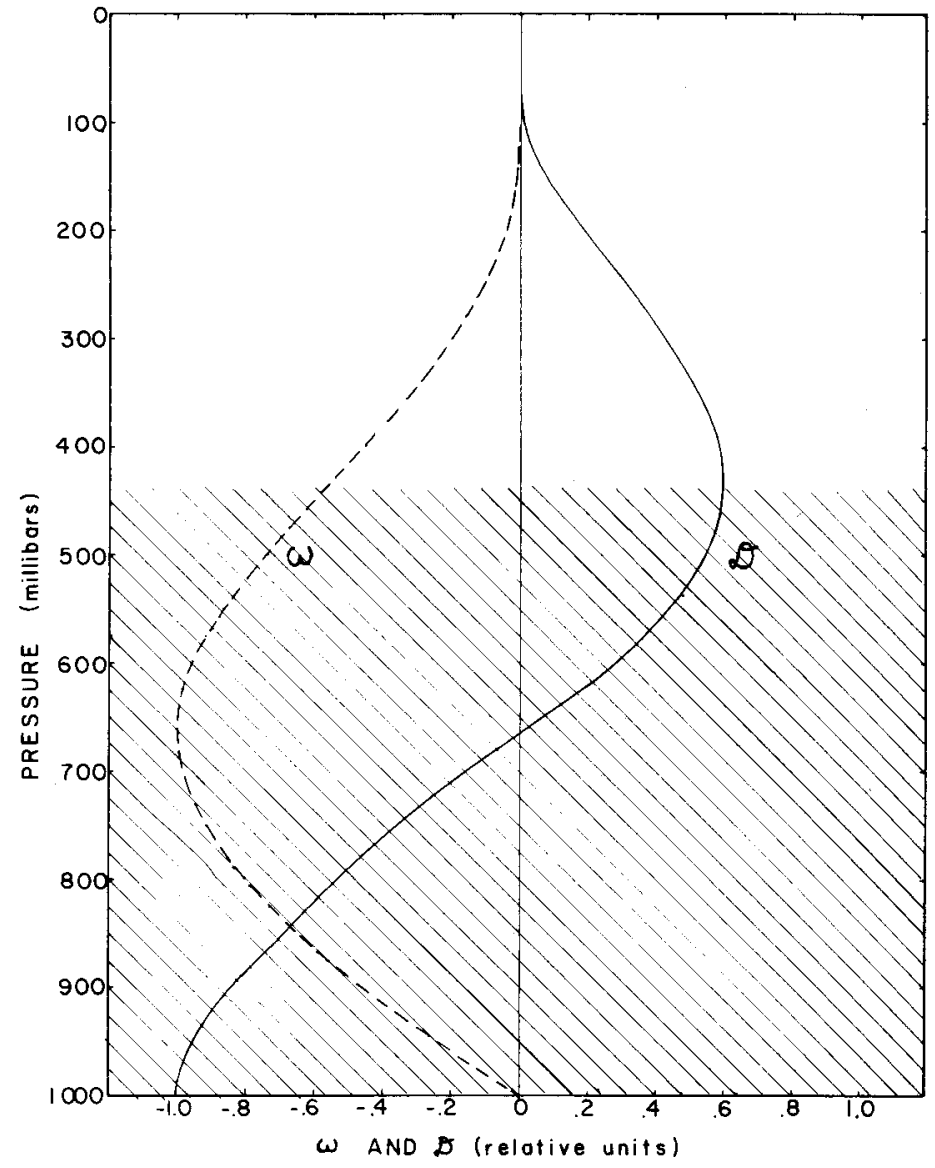

Figure 9.-Type III, case 2, vertical solutions. $\mathcal{D}$ (solid line), $\omega$ (dashed line), scale units normalized by maximum abscissae. Hatched area is conditionally unstable.

one boundary to the other. An initial guess of $\bar{\sigma}$ was made, and this was improved by means of Newton-Raphson iteration methods until both boundary conditions (8) and (12) were satisfied to sufficient accuracy. The method was checked by comparison of results for cases with known analytic solutions, such as case 1 of this section.

Figure 9 shows the solutions obtained for $\omega$ and $\mathcal{D}$. The unstable region is shaded, and above this region the disturbance amplitude rapidly decreases, becoming imperceptible in the stratosphere. The value of $\bar{\sigma}$ found for this solution is $-2.732 \times 10^{-3} \mathrm{~m}^{2} \mathrm{mb}^{-2} \mathrm{sec}^{-2}$. This result provides justification for the value of $-3 \times 10^{-3}$ used in evaluation of the stability criterion.

In comparing the sinusoidal vertical solutions of type I, equations (24)-(28) and figure 2 , with those of this section, we note that the sign of the geopotential disturbance reverses from low to high levels in both cases. This is generally true for any solution of the linear set (1)-(4) in which there is a reversal of the sign of divergence with height, since $\Phi$ is proportional to $\mathcal{D}$. This proportionality 
does not seem to accord with the true situation in welldeveloped tropical cyclones, which exhibit cyclonic rotation and negative disturbance geopotentials to great heights in their inner regions, and at the same time large negative values of relative vorticity and divergence.

In order to explain the disparity between the model and observations, attention is directed to the nonlinear terms of the motion equations. We show in a later section that those advective terms do seem to be responsible for extending the cyclonic wind field to higher levels as the disturbance amplitude becomes significant. In the very early stages the circulation reversal might be observed, but even then it could be masked if the disturbance developed within a preexisting circulation.

In order to further investigate this problem, one could set up perturbation equations on a basic nondivergent vortex. These equations become quite complicated when both horizontal and vertical shears are involved, and analytic solutions are out of the question for any physically reasonable cases. Rather than solve the perturbation equations by numerical methods, it would seem more profitable to solve the nonlinear equations directly, as an initial value problem. Berkofsky [1] has developed a numerical scheme for doing this. A deficiency of Berkofsky's model is that it excludes gravitational waves by means of a balance equation, and thereby eliminates the type of linear motions discussed in this paper.

\section{SURFACE FRICTIONAL EFFECTS}

If one assumes that a frictionally-forced updraft can be adequately expressed as a boundary condition of the form of (13), then this boundary condition may be used, in place of (12), to determine coefficients of the elementary solutions of (18). The resulting transcendental may be used, as with the type III solutions, to define an effective static stability, $\bar{\sigma}$, which satisfies the wave equation. The definition may be written as:

$$
\frac{\tan \left[(\sigma / \bar{\sigma})^{1 / 2} \pi p_{\mathbf{F}} / p_{0}\right]}{(\sigma / \bar{\sigma})^{1 / 2} \pi}=-\frac{q^{2}+f^{2}}{q f} B \frac{p_{0}-p_{\mathbf{F}}}{p_{0}}
$$

where we assume that $0<B<1$ for $q<f$ and $-1<B<0$ for $q>f$, and $p_{\mathbf{F}} \approx 900 \mathrm{mb} .=0.9 p_{0}$. For large disturbances, where $q \ll f$, the argument of the tangent approaches $\pi / 2$. Therefore $(\bar{\sigma} / \sigma)^{1 / 2} \approx 2$ and the largest unstable disturbance diameter is nearly doubled. On the other hand small disturbances with $q \gg f$ lead to values of $(\bar{\sigma} / \sigma)^{1 / 2}<1$.

\section{ENERGY RELATIONS}

It is of interest to consider the energy transformations and transports in our linearized system. The kinetic energy equation, obtained by dot multiplication of (1) by $\mathrm{V}$, may be written as follows:

$$
\frac{\partial \mathscr{K}}{\partial t}=-\nabla \cdot(\mathrm{V} \Phi)-\frac{\partial}{\partial p}(\omega \Phi)-\alpha \omega
$$

where $\mathscr{K}=\mathrm{V} \cdot \mathrm{V} / 2$. If (49) is integrated over all space, given external lateral boundary conditions as prescribed in section 3 , and $\omega$ vanishing at the upper and lower boundaries, the first two terms on the right vanish. After multiplying (4) by $\alpha / \sigma_{d}$, where $\sigma_{d}$ is the static stability for dry processes, we obtain an equation for available potential energy, similar to that defined by Lorenz [13].

$$
\frac{\partial \mathcal{A}}{\partial t}=\frac{\sigma}{\sigma_{d}} \alpha \omega
$$

where $A=\alpha^{2} / 2 \sigma_{d}$.

The integrated sum of 149 ) and (50) vanishes for dry processes, so that $(-\alpha \omega)$ is clearly the transformation from available potential to kinetic energy for the dry process. For moist motions, where $\sigma=\sigma_{w}$, the term $\left(\sigma_{d}-\sigma_{w}\right) \alpha \omega / \sigma_{w}$ is evidently the transformation of latent heat to available potential energy and is positive when $\sigma_{w}$ and $\omega$ are negative and $\alpha$ positive.

In the type II and III solutions of this study the energy relations are complicated somewhat by transports across the internal boundaries. The complete budget has been evaluated for type II, case 1 , and shows several interesting features. If we assume $\left(\bar{\sigma} / \sigma_{w}\right)^{1 / 2}=0.3$, from table 2 , and express all quantities as multiples of $J$, the total released latent heat energy, the following results are obtained.

$$
\left.\begin{array}{ll}
\frac{\partial \bar{A}_{d}}{\partial t}=0.367 \mathrm{~J} ; & \frac{\partial \bar{A}_{w}}{\partial t}=0.206 \mathrm{~J} \\
\frac{\partial \overline{\mathscr{K}}_{d}}{\partial t}=0.366 \mathrm{~J} ; & \frac{\partial \overline{\mathscr{K}}_{w}}{\partial t}=0.061 \mathrm{~J}
\end{array}\right\}
$$

where $J=0.585\left(-\sigma_{w} / q\right) A^{2} e^{2 q t}$ and $\bar{A}_{d}=\frac{1}{L p_{0}} \int_{0}^{L} \int_{0}^{p_{0}} \mathcal{A}_{d} d p d x$

with other quantities similarly defined. Figure 10 shows the relationships of these various energy terms. We see that of the total energy released by latent heat in the moist region 26.7 percent remains within the moist region, 6.1 
percent as kinetic energy, and 20.6 percent as available potential energy. Of the 73.3 percent which is transported into the dry environment (that is, nearly all of the transformation term), half is released as available potential energy and half as kinetic energy. Kinetic energy represents 42.7 percent of the total production.

It is interesting to compare these results with estimates of the energy budgets in tropical and extratropical cyclones. Palmén and Jordan [18] have estimated that about 30 percent of the kinetic energy release in a steady state tropical cyclone occurs within the inner $2^{\circ}$ latitude, where most of the latent heat energy is released, the remaining 70 percent being released mainly in the $2^{\circ}-6^{\circ}$ ring. In our model the corresponding percentages are about 16 percent and 84 percent. Palmén and Jordan find, however, that the total kinetic energy released is only about $2 \frac{1}{2}$ percent of the total latent heat energy released, as compared to our 42.7 percent. Palmén [17] in his analysis of hurricane Hazel, found that the kinetic energy released during the period of transformation from a tropical to an extratropical cyclone was about 12.5 percent of the latent heat energy released, but he was unable to estimate the transformation of available potential to kinetic energy during this time.

It seems, therefore, that the mechanical efficiency of our model is considerably too great, as compared with real large-scale systems. This is due both to the neglect of dissipative terms in this development and to the considerable changes in dynamics as the real disturbance develops and loses both its static instability and its vertical symmetry, by means of vertical and horizontal advection.

\section{NONLINEAR EFFECTS}

It is recognized that the atmospheric phenomena dealt with in this study frequently attain an amplitude at which the nonlinear terms of the meteorological equations are substantially larger than some of the linear terms. This is certainly sufficient cause for an examination of the foregoing results to determine in what way they might be altered by inclusion of these nonlinear terms in the basic differential equations. One method of approximating the nonlinear effects is to substitute the linear solutions into the nonlinear terms of the complete equations and thereby obtain a second-order approximation to the true nonlinear solutions. This might be considered as the beginning of an iterative process, in which each solution generates the terms of a subsequent higher order equation. The existence and convergence of such solutions has been demonstrated by W. Malkus and Veronis [15] as applied to a Bénard convection cell problem. The process is tedious when carried out to higher orders. In fact the first iterative step for our system results in an inhomogeneous differential equation, probably only numerically solvable. We are able, however, to obtain information as to the relative orders of magnitude and general effects of the nonlinear terms.
If we regard convective disturbances as eddies within a larger-scale environment, the advective terms of the thermodynamic equation represent the eddy transport of heat. Thus from the linear solutions we may to some degree approximate the convective heat transport of the linear systems.

We define $V^{\prime}, \Phi^{\prime}, \omega^{\prime}$, and $\alpha^{\prime}$ to be the second-order variables appearing when our linear solutions are substituted into the nonlinear terms of the motion and heat equations. Equations for these variables may be written as follows:

$$
\begin{gathered}
\frac{\partial \mathbf{V}^{\prime}}{\partial t}+f \mathbf{k} \times \mathbf{V}^{\prime}+\nabla \Phi^{\prime}=-\mathbf{V} \cdot \nabla \mathbf{V}-\omega \frac{\partial \mathrm{V}}{\partial p} \\
\frac{\partial \Phi^{\prime}}{\partial p}+\alpha^{\prime}=0 \\
\nabla \cdot \mathbf{V}^{\prime}+\frac{\partial \omega^{\prime}}{\partial p}=0 \\
\frac{\partial \alpha^{\prime}}{\partial t}-\sigma \omega^{\prime}=-\mathbf{V} \cdot \nabla \alpha-\omega\left(\frac{\partial \alpha}{\partial p}+\frac{\alpha}{\kappa p}\right) .
\end{gathered}
$$

If solutions of (5) and any of the boundary conditions used in this study are substituted for the variables on the right side and the system reduced to an equation in $\omega^{\prime}$, it may be shown that the inhomogeneous (nonlinear) terms are eliminated except for the last one in (55), and (52)-(55) reduce to

$$
\left(\frac{\partial^{2}}{\partial t^{2}}+f^{2}\right) \frac{\partial^{2} \omega^{\prime}}{\partial p^{2}}+\nabla^{2}\left(\sigma \omega^{\prime}\right)=\nabla^{2}\left(\frac{\omega \alpha}{\kappa p}\right) .
$$

A particular solution of (56) may be formally written as follows, where $p^{\prime}$ and $p^{\prime \prime}$ are dummy variables.

$$
\begin{aligned}
\omega^{\prime}=\int_{0}^{\mathrm{p}} \int_{0}^{\mathrm{p}^{\prime}} \cos \left[\left(\frac{q^{2}+f^{2}}{4 q^{2}+f^{2}}\right)^{1 / 2}\right. & \frac{2 \pi}{p_{0}}\left(p-2 p^{\prime}\right. \\
& \left.\left.+p^{\prime \prime}\right)\right] \frac{\nabla^{2}(\omega \alpha / \kappa p)}{4 q^{2}+f^{2}} d p^{\prime \prime} d p^{\prime} .
\end{aligned}
$$

In order to show the general nature of the solutions of (52)-(55), we consider a somewhat simpler case. If the atmosphere were incompressible, the last term on the right sides of (55) and (56) would vanish. This assumption alters the static stability parameter, $\sigma$, in that $\kappa$ is assumed infinite. Other than this change in interpretation the nature of the perturbation equations and their solutions is entirely unchanged. In this case the particular solution for (57) is

$$
\omega^{\prime}=0 .
$$

This is not trivial, since the remaining terms on the right of (52) and (55) do not vanish. $\Phi^{\prime}, V^{\prime}$, and $\alpha^{\prime}$ are found to be the following:

$$
\Phi^{\prime}=\frac{\sigma}{4 q^{2}}\left\{\left[\omega^{2}+\frac{\sigma}{\bar{\sigma}}\left(\frac{L}{k} \nabla \omega\right)^{2}\right]-\frac{2 q^{2}-f^{2}}{q^{2}+f^{2}}\left[\omega^{2}+\left(\frac{p_{0}}{\pi} \frac{\partial \omega}{\partial p}\right)^{2}\right]\right\}
$$




$$
\begin{gathered}
\mathrm{V}^{\prime}=\frac{\sigma f}{4 q^{2}\left(q^{2}+f^{2}\right)} \mathbf{k} \times \nabla\left[\omega^{2}+\left(\frac{p_{0}}{\pi} \frac{\partial \omega}{\partial p}\right)^{2}\right] \\
\alpha^{\prime}=-\frac{\sigma}{4 q^{2}} \frac{\partial}{\partial p}\left[\omega^{2}+\frac{\sigma}{\sigma}\left(\frac{L}{k} \nabla \omega\right)^{2}\right] .
\end{gathered}
$$

For the perturbation cases exhibited previously $\alpha^{\prime}$ is a function of pressure only, the tangential velocity is a function only of the horizontal coordinate, and $\Phi^{\prime}$ is a sum of the integrals of these one-dimensional functions. In figure 11 are shown horizontal profiles of $v^{\prime}$ and the horizontally variable part of $\Phi^{\prime}$ and vertical profiles of $\alpha_{w}^{\prime}$ and the vertically variable part of $\Phi_{w}{ }^{\prime}$, where $\omega_{w}$ and $\omega_{d}$ are obtained from the perturbation solutions of type II, case 1 with slab symmetry. The vertically variable parts of $\Phi_{d}^{\prime}$ and $\alpha_{d}^{\prime}$ vanish identically.

We are now able to verify the statements made in previous sections about the probable effects of nonlinear terms. From (59) and figure 11 one sees that the tangential wind field is changed by a term which is cyclonic at all levels within the moist region $\left(\sigma_{w}<0\right)$, the vorticity of which has a positive maximum at the center. In the dry region there is a corresponding anticyclonic wind, but of a much smaller magnitude, proportional to $\left(-\sigma_{w} / \sigma_{a}\right)^{3}$ times that of the moist region. These terms evidently tend to bring the perturbation disturbance into closer agreement with tropical cyclone wind profiles.

The $\alpha^{\prime}$ field tends principally to lift the level of the maximum heating, thus destabilizing the upper levels and allowing the developing cyclone to extend to much greater heights than the $5-\mathrm{km}$. depth of normal conditional instability in the tropical troposphere. The $\Phi^{\prime}$ field is composed of two parts. The vertically variable component is the hydrostatic integral of $\alpha^{\prime}$ and leads to decreased geopotential at intermediate pressure levels, consistent with the increasing depth of the cyclonic portion of the disturbance. The horizontally variable component of $\Phi^{\prime}$ has its maximum amplitude at the center, with the sign in the moist region the same as the sign of $2 q^{2}-f^{2}$. Thus if the linear disturbance is small and rapidly growing, i.e., $2 q^{2}>f^{2}$, this term represents a filling tendency. If $0<2 q^{2}<f^{2}$, however, both the linear and nonlinear terms provide low-level deepening at the center. The latter inequality then appears to specify a rather narrow size range within which disturbances may continue to grow to significant amplitudes, and thus represents a second-order stability criterion. It is not clear whether or how an initial disturbance might alter its size or shape to adjust to this criterion, nor is it certain how the compressibility term in (56) might change matters, but the effect may have an important bearing on the observed bimodal distribution of convective disturbances.

We now make quantitative estimates as to orders of magnitude of the nonlinear terms. If $\sigma_{w}=-3 \times 10^{-3} \mathrm{~m} \cdot .^{2} \mathrm{mb} .^{-2}$ sec. ${ }^{-2}$, and $f=5 \times 10^{-5}$ sec. $^{-1}$, by comparison of $(59)-(61)$ with $(26)-(28)$ it may be shown that $\alpha^{\prime} / \alpha$ and $v^{\prime} / v$ are $541417-60-3$
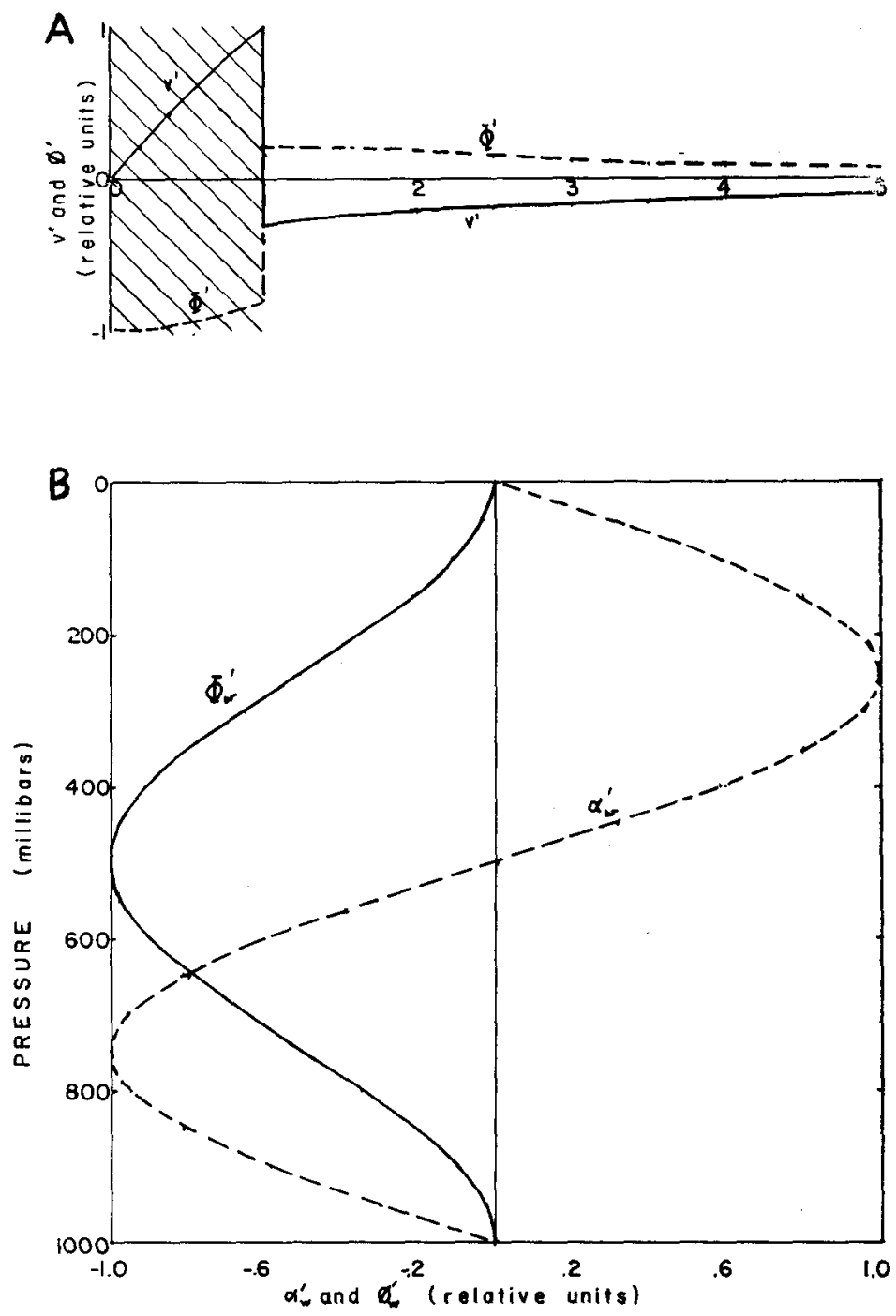

Figure 11.- Second-order components of nonlinear solutions for type II, case 1. (A) Horizontal profiles of $v^{\prime}$ (solid) and $\Phi^{\prime}$ (dashed). Moist region hatched. Scale units normalized by maximum ordinates. (B) Vertical profiles of $\boldsymbol{\Phi}^{\prime}{ }_{w}$ (solid) and $\alpha^{\prime}{ }_{w}$ (hatched). Scale units normalized by maximum abscissae.

approximately unity for $|\Phi|=300 \mathrm{~m}^{2}$ sec. $^{-2}$, or a surface pressure amplitude of about $4 \mathrm{mb}$. The first term of $\Phi^{\prime}$ is of the order of $|\Phi|$ when $|\Phi|=600 \mathrm{~m}^{2}$ sec. $^{-2}$, a pressure amplitude of $8 \mathrm{mb}$. The second term of $\Phi^{\prime}$ depends on its coefficient which may vary from -2 to +1 . If $q=f / 2$ this term is of the order of $|\Phi|$ when $|\Phi|=1500 \mathrm{~m} .^{2}$ sec. $^{-2}$, a surface pressure amplitude of $20 \mathrm{mb}$. Thus the secondorder stability criterion discussed above only becomes effective when the amplitude is substantial. At this stage the other nonlinear terms may have distorted the dynamics of the system so much that it is no longer applicable. In any case it is clear that the study of tropical cyclones by means of linear theory must be limited to rather small amplitudes.

With respect to meso-scale disturbances, the above amplitudes seem somewhat more liberal. A surface 
pressure disturbance of greater than $4 \mathrm{mb}$. is probably not greatly exceeded even in a squall line or hurricane convective band.

We now consider the nonlinear vertical heat transport by the linear disturbance solutions. In the present system heat energy is equal to $\left(c_{p} / R\right) \alpha p$.

After multiplication of $(61)$ by $\left(c_{p} / R\right) p$ and integration over a horizontal distance $X$, we obtain the expression for average vertical transport:

$$
\frac{\partial \bar{Q}}{\partial t} \approx \frac{c_{p}}{R} \frac{1}{X} \int_{0}^{x} p \frac{\partial \alpha^{\prime}}{\partial t} d x=-\frac{c_{p}}{R X}\left[\int_{0}^{x} p \frac{\partial}{\partial p}(\alpha \omega) d x+\left.p \alpha u\right|_{0} ^{X}\right.
$$

where the compressibility terms have again been neglected.

If $X$ is large or the disturbance is bounded, as in type II, case 2, the end-point term may be neglected. The term $\alpha \omega$ has opposite signs in the moist and dry regions but the integral is dominated by the contributions from the moist unstable region and is negative, with a maximum in the mid-troposphere. Thus for type II solutions $\partial \bar{Q} / \partial t \propto p \sin \frac{2 \pi p}{p_{0}}$; that is, a net transfer of heat from the lower to the upper levels.

\section{SUMMARY AND CONCLUSIONS}

Solutions to a complete set of linearized quasi-static meteorological equations under conditions of moist parcel instability have been obtained and investigated. The assumptions made, besides the usual perturbation requirements, included zero basic flow at all levels. constant Coriolis parameter, no orography, and upward motion always saturated and downward motion either completely unsaturated or completely saturated.

The solutions obtained are essentially internal gravitational waves, their dynamic stability criterion depending upon the horizontal and vertical scales of motion, the value of the Coriolis parameter, the static stability for dry and moist motions and its vertical variation, and surface frictional drag and/or forced ascent. Unstable motions exist for horizontal scales of motion smaller than a limiting radius of several hundred kilometers, and the maximum growth rate occurs for disturbances whose horizontal extent is about the same as, or smaller than, their height. The conclusion that cloud-scale motions grow more rapidly than those of meso- or cyclone-scale obviously does not preclude the existence of the latter, but relegates their explanation to methods not used in this study. It appears, further, that the smaller-scale motions, because of their compensating dry downdraft regions, tend to discourage development of larger-scale disturbances unless, or until an organized meso-scale or large-scale ascending core can be established by nonconvective processes.

A number of disturbance models, differing in the arrangement of moist and dry regions, boundary condi- tions, and the vertical static stability distribution were investigated, and the effects on dynamic stability expressed in terms of an effective static stability. It was found that the existence of a dry stable region with descending motions surrounding a moist ascending center (type II, case 1) decreases the effective instability in comparison with that of a disturbance where both ascent and descent are moist unstable. A dry downdraft or "eye" in the center (case 3) exerts little influence, while regions of bands of alternating moist updraft and dry downdraft (case 4) have effects midway between those of each of the two components. Motions are very rapidly damped above a tropopause or base of a stable layer (type III, cases 1 and 2), and the latter acts effectively as a solid surface.

The lower boundary condition used generally was that $\omega=0$ at $p=p_{0}$, but replacement of this condition by one more closely akin to the true atmospheric boundary condition had very little effect on results.

The effects of surface friction were evaluated in a rather crude fashion, and results indicate that instability is increased somewhat by this effect for cyclone-scale disturbances, and that the maximum possible disturbance size increases considerably.

It was shown that an internally self-consistent energy equation can be written in which, disregarding friction, the sum of the productions of kinetic and available potential energy is equal to the heat released by condensation. Analysis of this energy equation shows that most of the kinetic and potential energy is released in the outer descending region despite its origin in the heat released in the inner ascending region. The mechanical efficiency of the system was found to be several times higher than that of observed tropical cyclones.

Finally, the effects of nonlinear terms in the primitive equations and energy equations were considered. It was found that disturbances of cyclone scale are essentially nonlinear almost as soon as detectable, but meso-scale motions maintain their linear character somewhat longer. The nonlinear terms of the cyclone-scale disturbances tended to bring the linear disturbances into closer agreement with observed structure. Further, these nonlinear terms yield a second-order stability criterion which permits subsequent deepening of a small-amplitude disturbance when the horizontal dimensions are only slightly smaller than the critical (vanishing linear growth rate) size; that is, for disturbances of the observed scale of tropical cyclones.

The results of this study fully corroborate Malkus and Riehl's [14] recent work indicating that further significant progress toward solution of the problem of tropical cyclogenesis will require more detailed knowledge of the dynamics, thermodynamics, and perhaps hygro-kinematics of cloud-scale and meso-scale convection. The smallerscale convective processes tend to hinder cyclone development, both by the disruptive effects of dry downdrafts 
surrounding the updraft elements, and by the stabilization due to their nonlinear heat transports. These effects alone may be sufficient to limit development of tropical cyclones to areas and periods when strong forcing disturbances cause general ascent and moistening of a substantial area.

\section{ACKNOWLEDGMENTS}

The author wishes to express his sincere thanks to Prof. Seymour L. Hess for his valuable suggestions and criticisms, and to Drs. J. Smagorinsky and S. Manabe, whose suggestions and encouragements were exceptionally helpful. The research described in this paper was begun at Florida State Universtity with support by the U.S. Weather Bureau under Contracts Nos. Cwb-9395 and Cwb-9683 and was completed at the U.S. Weather Bureau, General Circulation Research Section.

\section{REFERENCES}

1. L. Berkofsky, A Numerical Model for the Prediction of Hurricane Formation, Unpublished thesis, Massachusetts Institute of Technology, 1957.

2. J. Bjerknes, "Saturated-Adiabatic Ascent of Air Through DryAdiabatically Descending Environment," Quarterly Journal of the Royal Meteorological Society, vol, 64, No. 275, Apr. 1938 , pp. $325-330$.

3. J. G. Charney and A. Eliassen, "A Numerical Method for Predieting the Perturbations in the Middle-Latitude Westerlies," Tellus, vol. 1, No. 2, May 1949, pp. 38-54.

4. A. Eliassen, "The Quasi-Static Equations of Motion with Pres-sure as an Independent Variable," Geofysiske Publikasjoner, vol. 17, No. 3, 1949, 44 pp.

5. F. Frankl, “On Cauchy's Problem for Partial Differential Equations of Mixed Elliptico-Hyperbolic Type with Initial Data on the Parabolic Line," Izvestiâ Akademii Nauk SSSR, Serita Matematicheskaia, vol. 8, 1944, pp. 195-224.

6. S. A. Gill, "A Process for the Step-by-Step Integration of Differential Equations in an Automatic Digital Computing Machine," Proceedings, Cambridge Philosphical Society, vol. 47, 1951, pp. 96-108.

7. C. L. Godske, T. Bergeron, J. Bjerknes, and R. C. Bundgaard, Dynamic Meteorology and Weather Forecasting, American Meteorological Society, Boston, Mass., 1957, 800 pp.
8. S. M. A. Haque, "The Initiation of Cyclonic Circulation in a Vertically Unstable Stagnant Air Mass," Quarterly Journal of the Royal Meteorological Society, vol. 78, 1952, pp. 394-406.

9. B. Haurwitz, "The Height of Tropical Cyclones and of the 'Eye' of the Storm," Monthly Weather Review, vol. 63, No. 2, Feb. 1935, pp. 45-48.

10. G. Hollmann, "Zur Frage der Lärmfilterung in den HydroThermo-Dynamischen Gleichungen," Beiträge zur Physik der Atmosphäre, vol. 31 , No. $1 / 2,1958$, pp. 5-30.

11. L. A. Hughes, "On the Low-Level Wind Structure of Tropical Storms," Journal of Meteorology, vol. 9, No. 6, Dec. 1952, pp. $422-428$.

12. C. L. Jordan, "Mean Soundings for the West Indies Area," Journal of Meteorology, vol. 15, No. 1, Feb. 1958, pp. 91-97.

13. E. N. Lorenz, "Available Potential Energy and the Maintenance of the General Circulation," Tellus, vol. 7, No. 2, May 1955 , pp. $157-167$.

14. J. Malkus and H. Riehl, "On the Dynamies and Energy Transformations in Steady-State Hurricanes," National Hurricane Research Project Report No. 31, 1959, 31 pp.

15. W. V. L. Malkus and G. Veronis, "Finite Amplitude Cellular Convection," Journal of Fluid Mechanics, vol. 4, 1958, pp. 225-260.

16. T. Ootani and H. Hatakeyama, "Upper Air Current in the Typhoon," Geophysical Magazine, vol. 6, 1932, pp. 97-122.

17. E. Palmén, "Vertical Circulation and Release of Kinetic Energy During the Development of Hurricane Hazel into an Extratropical Storm," Tellus, vol. 10, No. 1, Feb. 1958, pp. 1-23.

18. E. Palmén and C. L. Jordan, "Note on the Release of Kinetic Energy in Tropical Cyclones," Tellus, vol. 7, No. 2, May 1955 , pp. $186-188$.

19. N. A. Phillips, "Numerical Integration of the Primitive Equations on the Hemisphere," Monthly Weather Review, vol. 87, No. 9, Sept. 1959, pp. 333-345.

20. J. Smagorinsky, "On the Numerical Integration of the Primitive Equations of Motion for Baroclinic Flow in a Closed Region," Monthly Weather Review, vol. 86, No. 12, Dec. 1958, pp. $457-466$.

21. S. Syōno, "On the Formation of Tropical Cyclones," Tellus, vol. 5, No. 2, May 1953, pp. 179-195.

22. S. Syōno, Y. Ogura, K. Gambo, and A. Kasahara, "On the Negative Vorticity in a Typhoon," Journal of the Meteorological Society of Japan, Series 2, vol. 29, No. 12, Dec. 1951, pp. 397-414.

23. G. N. Watson, A Treatise on the Theory of Bessel Functions, 21 ed., University Press, Cambridge, Mass., 1944, 804 pp. 